\title{
Thermal Signature Measurements for Ammonium Nitrate/Fuel Mixtures by Laser Heating
}

\author{
Ashot Nazarian and Cary Presser* \\ National Institute of Standards and Technology
}

\begin{abstract}
Measurements were carried out to obtain thermal signatures of several ammonium nitrate/fuel (ANF) mixtures, using a laser-heating technique referred to as the laser-driven thermal reactor (LDTR). The mixtures were ammonium nitrate (AN)/kerosene, AN/ethylene glycol, AN/paraffin wax, AN/petroleum jelly, AN/confectioner's sugar, AN/cellulose (tissue paper), nitromethane/cellulose, nitrobenzene/cellulose, AN/cellulose/nitromethane, AN/cellulose/ nitrobenzene. These mixtures were also compared with AN/nitromethane and AN/diesel fuel oil, obtained from an earlier investigation. Thermograms for the mixtures, as well as individual constituents, were compared to better understand how the sample thermal signature changes with mixture composition. This is the first step in development of a thermal-signature database, to be used along with other signature databases, to improve identification of energetic substances of unknown composition. The results indicated that each individual thermal signature was associated unambiguously with a particular mixture composition. The signature features of a particular mixture were shaped by the individual constituent signatures. It was also uncovered that the baseline signature was modified after an experiment due to coating of unreacted residue on the substrate surface and a change in the reactor sphere oxide layer. Thus, care was required to pre-oxidize the sphere prior to an experiment. A minimum sample mass (which was dependent on composition) was required to detect the signature characteristics. Increased laser power served to magnify signal strength while preserving the signature features. For the mixtures examined, the thermal response of each ANF mixture was found to be different, which was based on the mixture composition and the thermal behavior of each mixture constituent.
\end{abstract}

\section{Key Words:}

Ammonium nitrate/fuel mixtures, laser-driven thermal reactor, oxidizer/fuel mixtures, thermal signatures, thermochemical behavior 
*Corresponding Author

Mailing Address:

National Institute of Standards and Technology

Bldg. 221, Rm. B310

100 Bureau Dr., Stop 8320

Gaithersburg, MD 20899-8320

Contact:

301-975-2612 (telephone)

cpresser@nist.gov (email) 


\section{Background}

Forensic identification of homemade explosive materials is essential for determining the composition and origin of the explosive mixtures and their precursors, and formulation procedures. Such information can then be used to develop strategies to disrupt or discourage the manufacturing of these materials, and provide protocols for their safe disposal. Homemade explosives are difficult to detect, and used increasingly by extremists and terrorists due to the widespread availability and easy accessibility of the precursors [1]. Currently, hundreds of compounds are classified as precursors $[2,3]$. The improvised nature of the mixture chemical composition and formulation/synthesis procedures presents a formidable challenge for forensic processing and analysis. Most common mixtures are prepared by physically mixing an oxidizer with a fuel, however in more recent years, improvised formulations have been developed through chemical reaction synthesis to combine both the oxidizer and fuel into complex molecules. In a recent FBI investigation [4], a variety of fuels were identified as significant materials to warrant further characterization of their explosive/detonation properties (e.g., detonation velocity, pressure/impulse, critical radius, etc.). Standard procedures were also developed for preparing these materials.

The explosive properties of ammonium nitrate (AN)/fuel mixtures has been investigated extensively. In a series of articles, Miyake et al. (e.g., $[5,6])$ investigated the detonation properties of AN/activated carbon mixtures for stoichiometric composition for different physical parameters (i.e., mixing ratio and charge diameter). They found that the detonation velocity at the peak pressure was far below the theoretically predicted value and thus confirmed its non-ideal detonation behavior (i.e., detonation reaction kinetics are relatively slow, resulting in a long detonation zone, and dependency on different physical parameters). Hurley et al. [7] studied the explosion characteristics of AN with different alternative solid fuels (i.e., sugar and coal dust). Grain size of the AN and solid fuel was found to be a critical parameter. The solid fuel detonation velocity was also found to be lower than that for fuel oil. Buczkowski [8] presents calculated values for the heat of explosion (total thermal energy release) for isolated AN, as well as for AN/fuel (ANF) mixtures, which are compared with those for standard high explosives such as trinitrotoluene (TNT) and hexogen. The addition of fuel to isolated AN was found to increase considerably the heat of explosion, while inert substances tend to absorb and reduce the released thermal energy [9]. Their calculated result for isolated AN was $1.712 \mathrm{~kJ} \cdot \mathrm{g}^{-1}$, which compared well with the total energy release measured by Nazarian and Presser [10] of $2.10 \pm 0.22 \mathrm{~kJ} \cdot \mathrm{g}^{-1}$. The measured thermal decomposition temperature for both studies was estimated to be $503 \mathrm{~K}$. It is noted that AN mixtures with wooden dust, coal, and mineral oil are characterized by a more than two-fold increase in the heat of explosion (i.e., $3.803 \quad \mathrm{~kJ} \cdot \mathrm{g}^{-1}, \quad 3.860 \quad \mathrm{~kJ} \cdot \mathrm{g}^{-1}, \quad$ and $4.018 \mathrm{~kJ} \cdot \mathrm{g}^{-1}$, respectively), as compared to isolated AN. These values are only lower than the value for TNT by $10 \%$ to $20 \%$ (i.e., $4.584 \mathrm{~kJ} \cdot \mathrm{g}^{-1}$ ), whereas, the AN/aluminum mixture value (i.e., $6.744 \mathrm{~kJ} \cdot \mathrm{g}^{-1}$ ) is significantly higher than that of TNT. Also, the thermal decomposition temperature was found to decrease with the additive fuels, as compared to the isolated AN. 


\subsection{Need for Improving Confidence in Identifying Mixture Composition}

A variety of commercially available analytical techniques are used in forensics analyses to identify the composition of improvised mixtures to some level of uncertainty [11]; often the compound signature may not be in the instrument database, forcing the software to make a "best" choice [12]. These techniques provide thermal, mass, and optical-spectral signatures from the unknown composition for comparison to signatures in database libraries [13]. In addition, signatures from secondary sources (e.g., residues from either materials used in the preparation or products of reaction) potentially can provide additional information for identification purposes. To improve the confidence level for identifying a particular mixture and its precursors, and meet forensic-analysis standards, new methodologies and database tools are required to statistically combine signatures from a variety of analytical instruments for chemical identification under different environmental conditions. Statistically combined signatures obtained from different independent measurement techniques (as opposed to reporting these signatures separately), can potentially improve the confidence level for identification and will reduce the forensic processing time. A statistical/probability methodology is needed to combine (fuse) accessible signature data sets [14], which specifies confidence levels associated with the proper identification of a selected chemical. A variety of different mathematical approaches exist (e.g., Bayesian statistics [15], Dempster-Shafer theory $[16,17]$, and Rule-Based Data Fusion [18] methods), which may be used to address this issue of statistical data fusion. Ultimately, the thermal, mass, and spectral signatures can be correlated to pre-identified precursors, and used to establish a database with improved confidence levels. Thus, measuring thermal, mass, and spectral signatures of a precursor or residue of unknown composition could then be matched with signatures of known composition from a variety of databases, with improved confidence levels through the use of statistical data fusion, to identify the unknown substance with higher certainty. To this end, this investigation addresses one aspect of this overall strategy, which is to provide thermal signatures from calorimetry measurements.

\subsection{Issues Associated with Commercially Available Calorimetry Techniques}

Typically, calorimetry techniques are used to estimate the thermal energy release from materials. Commercially available calorimetry techniques include differential scanning calorimetry, differential thermal analysis, thermogravemetric analysis, and a family of variant

arrangements. These systems are widely used in a variety of different applications, however, one must be aware of the reported systemic operating issues (affecting sensitivity and resolution [19]), as well as some more significant limitations. For example, these techniques are associated with relatively low heating rates (limited to $10 \mathrm{~K} / \mathrm{min}$ to $20 \mathrm{~K} / \mathrm{min}$ ), which can result in integration of the detailed thermal behavior, as fuel volatile components decompose and vaporize prior to reaching the sample preselected steady-state temperature [20]. The sample composition then may not be representative of the expected thermal behavior. Thus, higher heating rates are required to attain higher temperatures before the occurrence of 
significant substance decomposition and chemical reactions. Also, the instrument measurement depends on sample size and heating rate. If the sample mass is too small, or the heating rate too high (as with micro-differential scanning calorimetry [21]), then the sample is consumed before completion of the thermal cycle and precise thermogram details are not recorded with actual sample. The data obtained with these instruments may underestimate the explosive potential of an energetic material under certain conditions [20,22]. In addition, multiphase mixtures and nonhomogeneous solutions [19, 23] are unsuitable for instrument analysis due to endothermic vaporization of volatile components (preferential vaporization) and leakage from the pan [23]. Other reported effects [24] include sensitivities due to mass contact area, geometry and shape as it sits in the pan substrate. Also, inert gas flow carries away reaction byproducts, which may also provide important forensic information.

\subsection{Methodology for this Investigation}

Reliable signature data can enable law enforcement and military agencies to reduce threats by identifying energetic material composition and formulation procedures. To this end, this investigation focused on measuring the thermal signature for several ANF mixtures and associating the features of the ANF thermal signature to its mixture composition. A developing laser-heating technique, referred to as the laser-driven thermal reactor (LDTR), was used because it can address several of the issues associated with the commercially available calorimetry techniques [20]. Some of the advantages include: 1) an order of magnitude higher heating rate and larger sample mass capability to capture the thermal behavior of volatile constituents, thus allowing application to nonhomogeneous, multicomponent, multiphase samples, 2) direct laser heating that enables measurement of the total energy loss due to both thermal and chemical heat release; there is no single conventional technique available that provides both thermochemical properties, and chemical kinetic rates and reactions [25], 3) laser heating of the reactor sphere eliminates convective heating of the surrounding gases under reduced pressures (heating is essentially by radiation and conduction), 4) analyses can be carried out in different surrounding environments (e.g., inert, pyrolytic, oxidative), and 5) sampling of decomposition/reaction gaseous byproducts enables species analysis by gas chromatography/mass spectrometry (not carried out in this investigation). Table 1 lists the ANF mixtures and precursors studied in this investigation. The ratio given for each material is the stoichiometric oxidizer/fuel mass fraction. In an earlier investigation [10], quantitative results for ammonium nitrate (AN) and nitromethane (NM) were presented under a variety of operating conditions (i.e., changing mass and heating rates) and preparation protocols. Ammonium nitrate/nitromethane (ANNM) mixture and its individual constituents, as well as ammonium nitrate/No. 2 diesel fuel oil (ANDF) were also investigated under a variety of operating conditions (i.e., fuel aging) and preparation protocols $[20,25]$. This investigation further expands the analysis of ANF to identifying thermal behavior and signature subtleties associated with mixture composition for an additional ten mixtures. The oxidizer for most of the mixtures was ammonium nitrate (AN). The liquid fuels selected for this investigation were diesel fuel oil, kerosene, and ethylene glycol. Solid fuels included confectioner's sugar and 
cellulose (tissue paper), petroleum jelly, and paraffin wax. Nitromethane and nitrobenzene served as either a liquid fuel or an oxidizer.

\section{Experimental}

\subsection{Brief facility description}

The LDTR experimental facility and theory of analysis are detailed in Nazarian and Presser $[10,20,25]$. The pertinent information regarding the experimental apparatus is summarized for clarity, along with a description of the current facility modifications for these experiments. The laser-driven thermal reactor consists of a sphere-shaped, gold-plated, copper-foil reactor (with an outer diameter of $(18.20 \pm 0.03) \mathrm{mm}^{1}$ and thickness of $(0.140 \pm 0.001) \mathrm{mm}$ mounted within a vacuum chamber, along with optical, gas supply, and computer-controlled data acquisition subsystems (see Figs. 1 and 2). Liquid/powdered mixture samples were placed in a gold-plated copper pan substrate and supported on a customized K-type fine-wire 'sample' thermocouple $(0.25 \mathrm{~mm}$ bead diameter, unsheathed, and $0.200 \mathrm{~s} \pm 0.002 \mathrm{~s}$ response time) stationed at the center of the reactor (defining the 'sample temperature'). The expanded uncertainty for the temperature was $2.4 \mathrm{~K}$, including the Type B uncertainty of $1.2 \mathrm{~K}$. The pan mass

was $(55.5 \pm 0.1) \mathrm{mg}$ with dimensions of $(4.950 \pm 0.001) \mathrm{mm}$ (inner diameter), $(2.340 \pm 0.001) \mathrm{mm}$ (height), and $(0.1250 \pm 0.0006) \mathrm{mm}$ (thickness). Other pan materials have been used instead of copper to remove possible reaction of the sample with the pan, but this was not the case for these experiments. A second 'reactor' thermocouple was flush with the inside surface of the reactor wall (defining the 'reactor temperature'). The reactor assembly is heated from opposing sides by an infrared Nd:YAG laser beam (operating at a wavelength of $1.064 \mu \mathrm{m}$ ) to achieve nearly uniform sample temperature. The laser power (with an expanded uncertainty of better

than

$1.0 \%$ of the mean laser power) was considered steady during an experiment and unchanged from run-to-run. Effects due to the laser beam intensity distribution over the copper reactor sphere are considered insignificant because 1) the laser beam is set to cover a large portion of the sphere's surface, 2) copper has a relatively high value for thermal conductivity $\left(401 \mathrm{~W} \cdot \mathrm{m}^{-1} \cdot \mathrm{K}^{-1}\right.$ at $\left.300 \mathrm{~K}[26], 3\right)$ relative dimensions of the sample and thin-walled sphere, and 4) thermal radiation is the dominant mode of heat transfer at high temperature between the interior sphere surface and the sample. The temperature uniformity of heating a sample placed within the center of the sphere (for a volume with dimensions of about $10 \mathrm{~mm}$ diameter by $5 \mathrm{~mm}$ height) is of the order of $1 \mathrm{~K}$. Sample/pan alignment with the thermocouple at the center of the sphere was estimated to be within $1 \mathrm{~mm}$. The reactor is stationed within the vacuum

\footnotetext{
${ }^{1}$ Estimation of the measurement uncertainty for this study is determined from statistical analysis of a series of replicated measurements (referred to as Type $A$ evaluation of uncertainty), and from means other than statistical analysis (referred to as Type B evaluation of uncertainty) [27]. The Type A uncertainty is calculated as $k u_{c}$, where $k$ is the coverage factor and $u_{c}$ is the combined standard uncertainty. The value for $u_{c}$ is estimated statistically by $s n^{-1 / 2}$, where $s$ is the sample standard deviation and $n$ is the number of samples. For $n=50, k=2.01$, representing a level of confidence of $95 \%$.
} 
chamber that allows for control of the environment (e.g., gas pressure and composition) around the sample. During an experiment, the sample and reactor temperatures were recorded with respect to time (referred to as 'thermograms') by the data acquisition system and then processed for the specified thermochemical and thermo-physical information of interest. The data-acquisition sampling rate is $1 \mathrm{MHz}$, which is averaged and reported every $0.25 \mathrm{~s}$ because of the limited thermocouple response time. There are no issues regarding explosion of the ANF mixtures because of the small quantities that are used for the measurements. Fumes from an experiment are exhausted from the chamber to avoid inhalation of any reactive fumes.

Experiments were carried out to obtain a 'baseline thermogram' (i.e., thermogram measurements with the pan substrate and no sample), and then repeated with the sample mixture ('sample thermogram'). Regarding the thermogram signal-to-noise ratio, one can estimate the noise level from the baseline thermograms. The expanded uncertainty of the reactor and sample temperatures for repeated baseline and sample thermograms (considering the absolute difference in temperatures per unit time, $\Delta T$, of two replicated thermograms) was calculated to be better than $1 \mathrm{~K} ; \Delta T$ generally is less than $10 \mathrm{~K}$ with a percent difference of less than $5 \%$ ). Measurements were carried out in nitrogen under a reduced pressure of $4 \mathrm{kPa}$, which eliminated thermal convection within the reactor sphere, and thus conduction and radiation were the dominant modes of heat transfer. The inert gaseous environment also isolated the fuel and oxidizer from a more complex oxidative environment of the surroundings (i.e., in the presence of the ambient air). Calculation and observation have indicated that evacuation of the chamber two to three times is sufficient to replace the ambient air with the inert gas. Experimental conditions were chosen by initially selecting nominal values for the laser power setting (LS1 - lower setting with a total output power of about $69 \mathrm{~W}$, and LS2 higher setting with about $134 \mathrm{~W}$ ) and the sample mass (i.e., $1 \mathrm{mg}$ to $5 \mathrm{mg}$ ). Minimum settings were used at the start to preserve the copper reactor sphere from chemical corrosion by the ANF mixtures. Experiments were carried out to obtain the 'sample thermal signature', being defined to include both the sample thermograms (i.e., with both the sample and reactor temperatures) and 'sample derivative profiles' (i.e., variation of the sample temperature-time derivative, $d T / d t$, with sample temperature, $T$, for the sample thermograms). Similarly, one can define the 'baseline thermal signature' along with the 'baseline derivative profiles' (i.e., $d T / d t$ versus $T$ for the baseline thermogram). Changes in the baseline or sample derivative profiles are solely due to variations in the slope (estimated by differencing adjacent points per time interval) of the respective thermogram (there is no independency). The 'heating rate' of the system responding to the laser heating is defined by the maximum derivative of the baseline derivative profile, using the sample temperature. Note that the laser power can be fixed while the heating rate (as defined) be different for two experiments because of systemic modifications (e.g., incorporating a new reactor sphere, change in the oxide layer on the sphere surface, change in the laser power). So the heating rate and not the laser power is the relevant parameter of interest. If the sample derivative profile was featureless then the heating rate 
and/or mass were increased and the experiments repeated to enhance the profile features and facilitate comparison of the thermal signatures with the other ANF mixtures. The strength of the various peaks associated with each ANF mixture was not optimized, but the laser power level was changed to a second preset setting to enable detection of the signature peaks. Note that increasing the laser power setting increased both the heating rate and maximum achieved temperature. When 'profile features' (i.e., values of significant magnitude above the profile threshold) were observed in the sample derivative profile, then these operating conditions were selected for the sample mixture. Baseline measurements were also carried out before and after experiments to check for unwarranted changes in the system and to facilitate the determination of the mixture endothermic/exothermic behavior.

The ingredients for the ANF mixtures were over-the-counter products, as would be acquired by terrorists. Particle morphology and variability were not characterized for the powdered materials.

\subsection{Brief theoretical description for quantitative evaluation of thermograms}

\subsubsection{Thermal Energy Balance}

As mentioned above, the details of the theoretical analysis [10, 20, 25], as well as results for AN, NM, ANNM, and ANDF, provide examples of the quantitative information that can be obtained with the LDTR technique (noting again that this investigation focused on the signature features). The following summarizes briefly how one can determine sample endothermic/exothermic behavior, specific heat release rate, and total specific heat release from the thermograms. The theoretical model is based on a representation of the heating process associated with the above-mentioned experimental arrangement. The following thermal energy balance governs the heating process:

$$
m c_{p}(T) d T / d t=I_{I} A \beta(T, \lambda)-F\left(T, T_{o}\right)+\Delta m(t) q(T)
$$

where the rate of change of sample internal thermal energy is given by the term on the left side of Eq. $1, T$ is the sample temperature, $c_{p}(T)$ is the sample specific heat capacity at the sample temperature, and $m$ is the sample initial mass. The first term on the right side of the Eq. 1 is the thermal energy absorbed by the sample, where $I_{I}$ is the laser beam incident radiation intensity that heats the sample, $A$ is the sample geometric cross-sectional area, $\beta(T, \lambda)$ is the sample hemispherical absorptivity at temperature $T$ and laser wavelength $\lambda$. The heat transfer term, $F\left(T, T_{o}\right)$, represents the sample thermal energy losses due to conduction, convection, and radiation throughout the arrangement. The parameter $T_{o}$ is a reference temperature of the surrounding environment, which for this study is the sample temperature of the baseline. Thermal energy losses due to chemical reaction and vaporization are considered if there is a 
detectable mass change from the initial value, $\Delta m(t)$, with respect to time $t$ after heating the sample. The term $q(T)$ is defined as the specific heat release rate due to chemical reactions (i.e., the rate at which thermal energy is released or absorbed by a substance per unit mass during chemical reactions).

\subsubsection{Thermal analysis}

The protocol used for these experiments involves heating the reactor to a steady-state temperature (referred to as the 'heating-rate' approach) [20]. For this approach, the sample and reactor temperatures (i.e., for the baseline without sample) are recorded for two runs at two different laser fluences, see Refs. $[20,25]$. The temperature-dependent relaxation time $\tau$ (also referred to as the heat transfer parameter) is given by:

$$
\tau(T)=\frac{T_{2}-T_{1}}{\left(\frac{d T}{d t}\right)_{1}-\left(\frac{d T}{d t}\right)_{2}}
$$

where $T_{1}$ and $T_{2}$ are the baseline temperatures (i.e., with only the copper substrate and without the sample) for the two different laser fluences at time $t_{1}$ and $t_{2}$, respectively, which correspond to the same reactor temperature. The reactor temperature is chosen arbitrarily at a value with sufficient sensitivity to determine the baseline temperature-time derivatives, $(d T / d t)_{1}$ and $(d T / d t)_{2}$, which correspond to $T_{1}$ and $T_{2}$, respectively.

Once the relaxation time is known, a set of experiments is carried out at a chosen laser fluence (corresponding to a preselected sample steady-state temperature), i.e., one thermogram with only the substrate (baseline), and a second with the sample and substrate. The equations representing these two experiments are:

with both the sample and substrate

$$
m c_{p}(T) d T / d t=I_{I} A \beta(T, \lambda)-F\left(T, T_{o}\right)+\Delta m(t) q(T)
$$

without the sample (substrate only)

$$
m c_{p}(T) d T / d t=I_{I} A \beta(T, \lambda)-F\left(T, T_{o}\right)
$$

We assume that $m_{s a s} \approx m_{s o} \equiv m, c_{p, s a s} \approx c_{p, s o} \equiv c_{p}$, and $\beta_{s a s} \approx \beta_{s o} \equiv \beta$, since the mass of the sample is considered small compared to the substrate mass. The subscript 'sas' refers to the combined sample and substrate in Eq. 3 and the subscript 'so' refers to the substrate only in Eq. 4. Subtracting Eq. 4 from Eq. 3, substituting into Eq. 2, and rearranging terms results in the following expression for the specific heat release rate, $q(T)$, due to chemical reaction [25]: 


$$
q\left(T_{s a s}\right)=\frac{m c_{p}\left(T_{s a s}\right)}{\Delta m(t)}\left[\frac{T_{s a s}-T_{s o}}{\tau}+\left.\frac{d T}{d t}\right|_{s a s}-\left.\frac{d T}{d t}\right|_{s o}\right]
$$

If $q(T)<0$ then the thermal behavior for that part of the heating process is endothermic and if $q(T)>0$ then the behavior is exothermic. Integration of Eq. 5 with respect to time for the entire experiment (including endothermic and exothermic processes) results in an expression for the total specific heat release, $Q=\int q(T) d t=-\Delta H$ (where $\Delta H$ is the change in enthalpy of a chemical reaction) $[20,25]$. One can then compare the measured change in enthalpy to calculated values, which are derived from the set of possible overall chemical reactions (i.e., as obtained from the literature). The most probable reaction is assumed to be the one with a calculated value of $\Delta H$ that is similar to the measured value [20].

\subsection{ANF precursor Preparation Procedures}

In this investigation, all of the ANF mixtures were recommended by collaborating forensic experts and formulated according to established recipe procedures [28]. The procedures used in preparing the different mixtures are described below.

2.3.1. Ammonium nitrate/nitromethane (ANNM); Ammonium nitrate/diesel fuel oil (ANDF): Both ammonium nitrate/nitromethane (ANNM) mixtures and ammonium nitrate/No. 2 diesel fuel oil mixtures (ANDF) were formulated, as described in Ref. [28]. In general, mixtures were prepared by dripping fuel onto AN particles with a syringe until the required stoichiometric value was ascertained with repeated mass measurements on an electronic precision balance. The mixture has the texture of a moist aggregated sediment.

2.3.2. Ammonium nitrate/kerosene (ANK); Ammonium nitrate/ethylene glycol (ANEG): Kerosene 1-K $\left(K_{1}\right)$ for heaters, lanterns, and stoves, and ethylene glycol (EG) were procured from a local hardware store. The same procedure for ANDF was used to prepare these mixtures, i.e., the fuel was dripped onto the solid oxidizer ammonium nitrate (AN) and weighed on an electronic mass balance to obtain the required mass fraction.

2.3.3. Ammonium nitrate/paraffin wax (ANPW): Paraffin wax (PW) is the common name for the alkane hydrocarbons with the formula $\mathrm{C}_{n} \mathrm{H}_{2 n+2}$ with range from $\mathrm{C}_{20} \mathrm{H}_{42}$ to $\mathrm{C}_{40} \mathrm{H}_{82}$. It is available in many drug store products and directly from candles [29]. Paraffin wax (melting point is $320 \mathrm{~K}-338 \mathrm{~K}$ ) was mixed with AN by melting the wax in a water bath, and then gradually adding AN (with a melting point of $443 \mathrm{~K}$ ) to the melted PW while continuously stirring the melt. The mixture was then cooled down to room temperature, placed on a substrate, and weighed to obtain the required mass fraction. 
2.3.4. Ammonium nitrate/petroleum jelly (ANPJ): Petroleum jelly (PJ) is available over-thecounter in drug stores (e.g., Vaseline ${ }^{2}$ ) and other products. It may be substituted for vegetable ghee (e.g., margarine, Vanaspati, or vegetable fat). The chemical formula is $\mathrm{C}_{n} \mathrm{H}_{n+2}$ from $\mathrm{C}_{20} \mathrm{H}_{42}$ to $\mathrm{C}_{40} \mathrm{H}_{82}$. The PJ was melted in a water bath, and $\mathrm{AN}$ was gradually added to the PJ, while continuously stirring the mixture. The mixture was then cooled down to room temperature. A sample was then placed on a substrate and weighed on the electronic mass balance to obtain the required mass fraction.

2.3.5. Ammonium nitrate/confectioner's sugar (ANS): Confectioner's sugar, also known as powdered sugar or icing sugar, has fine sugar particles that contain a small amount of anticaking agent. It is made by milling normal granulated sugar into a powdered state. Powdered sugar is generally mixed with cornstarch to avoid coagulation and lump-formation effects. "Domino" powdered sugar was used for these experiments. Ammonium nitrate and confectioner's sugar were added to a glass vial and mixed for one minute with a glass rod to obtain the required mass fraction.

2.3.6. Ammonium nitrate/cellulose (ANC): The cellulose used in these experiments was tissue paper $\left(\mathrm{C}_{\mathrm{TP}}\right)$. According to the literature [30], the composition of tissue paper is cellulose $\left(\mathrm{C}_{6} \mathrm{H}_{10} \mathrm{O}_{5}\right)$ and calcium carbonate $\left(\mathrm{CaCO}_{3}\right)$. Tree bark is the source of the paper. Cellulose in the tree bark has strong fibers that keep the tissue from clumping and forming pulp. Calcium carbonate is added to strengthen and maintain the shape of the cellulose fibers. Based on unpublished ATF studies [31], the cellulose used for these experiments was "Charmin" doubleply tissue paper.

Cellulose samples were cut in $4 \mathrm{~mm}$ circular disks. Each disk was weighted and the average mass was $(1.075 \pm 0.050) \mathrm{mg}$. A cellulose disk was placed in a glass dish and a prepared AN/water solution was dripped entirely over the cellulose surface with a pipette to obtain the required mass fraction. For a larger sample mass, additional cellulose disks (layers) were added followed by additional AN/water solution. The sample was then left to dry (resulting in removal of the water), and re-weighed.

2.3.7. Nitromethane/cellulose (NMC); Nitrobethane/cellulose (NBC): The cellulose sample was prepared as described above for ANC. The cellulose sample was placed on a disk-shaped substrate and either NM or NB was added by pipet until saturation (i.e., completely immersed in liquid), simulating the prescribed recipe procedures. The sample was then allowed to dry in the ambient over a period of a few days. No mass fraction is reported as a result of saturating the cellulose.

\footnotetext{
${ }^{2}$ Certain commercial equipment or materials are identified in this publication to specify adequately the experimental procedure. Such identification does not imply recommendation or endorsement by the National Institute of Standards and Technology, nor does it imply that the materials or equipment are necessarily the best available for this purpose.
} 
2.3.8.

Ammonium

nitrate/cellulose/nitromethane

(ANCNM);

Ammonium

nitrate/cellulose/nitro-benzene (ANCNB): Cellulose sample was prepared as for the abovementioned ANC case. The ANC sample was placed on a disk-shaped substrate and NM or NB was added by pipet until saturated. Again, no mass fraction is reported as a result of saturating the cellulose.

\section{Results and Discussion}

The experiments are grouped in the following order for comparison of compositions (see Table 1), i.e., ammonium nitrate/fuel oil (ANFO) mixtures for liquid and then solid fuel components (ANDF [25], ANK, ANEG, ANPJ, ANPW, ANS and ANC), C TP cases (ANC, NMC, and NBC), and three-component mixtures (ANCNM and ANCNB). Table 1 also presents the ANF mixture stoichiometric oxidizer/fuel mass fraction, initial sample mass, and heating rate. The terms LS1 and LS2 represent a lower laser power setting at a total output power of about $69 \mathrm{~W}$, and higher setting at about $134 \mathrm{~W}$, respectively. These two laser power settings represent nominal settings for the heating rate, as described above; the signal strength was not optimized for each thermogram, but the laser power level was changed to the second preset setting to enable detection of the various signature peaks. The condition of the reactor sphere after each experiment (which eventually degrades with repeated laser heating), or replacement with a new sphere, will influence the heating rate, and thus the baseline thermogram was obtained prior to each sample run.

Results were also available for ANNM [25] and the individual constituents AN [10, 25], $C_{\text {TP }}$, and NM [10] (NB data were unavailable) for comparison to and elucidation of the ANF mixture thermogram features. The operating conditions (i.e., reduced pressure in nitrogen) for the single-component experiments were the same as that for the mixtures. The initial mass and heating rate was $(1.42 \pm 0.1) \mathrm{mg}$ and $75 \mathrm{~K} \cdot \mathrm{s}^{-1}(\mathrm{LS} 2)$ for AN, $(0.58 \pm 0.1) \mathrm{mg}$ and $23 \mathrm{~K} \cdot \mathrm{s}^{-1}$ (LS1) for $\mathrm{NM}$, and $(1.0 \pm 0.1) \mathrm{mg}$ and $68 \mathrm{~K} \cdot \mathrm{s}^{-1}$ (LS2) for $\mathrm{C}_{\mathrm{TP}}$, respectively. Note that although the liquid component is continually evaporating, the time between sample preparation and the experiment was deemed short (i.e., within $30 \mathrm{~s}$ ) and of negligible effect (i.e., on changing in sample mass). The forthcoming figures present the sample thermograms and sample derivative profiles for both the baseline and sample experiments. The difference between the sample and baseline temperatures provides information about the sample thermal behavior $[10,20,25]$. If the sample thermogram is above the baseline then the sample behavior is exothermic, while if below the baseline thermogram its behavior will be endothermic. The baseline and sample thermograms are matched (with regard to the experiment time) so that the initial rise in temperature overlay with one another (assuming that the initial sample temperatures are similar). As discussed in Refs. [10, 20, 25], this information, along with the sample derivative 
profiles, can be used to obtain the sample total thermal release; however, the focus of this study was on the sample thermal signature characteristics. In particular, of interest was the relationship between the measured sample thermal signature and ANF mixture composition. To this end, features developed by the sample derivative profile are presented along with the baseline derivative profile for the sole purpose of highlighting the features for the sample thermal signature, as opposed to the relatively featureless baseline thermal signature. However, several issues developed during this investigation regarding the baseline thermogram measurement and its potential effect on evaluation of the sample thermal behavior, which are detailed in the next section.

\subsection{Issues regarding measurement of the baseline}

In earlier LDTR investigations, single-component samples were studied under reducing conditions, which involved only thermal decomposition of the sample. In more recent investigations, experiments were carried out with mixtures (with one constituent being an oxidizer), resulting in the occurrence of chemical reactions, release of vapors from the chemical byproducts, and residue remaining in the pan after reactions were completed. As mentioned earlier, knowledge of the baseline is important for determining the thermochemical characteristics (i.e., endothermic/exothermic behavior, heat release rate, and total heat released) because these parameters are dependent on the temperature difference between the sample and baseline thermograms. One expects that the system (sphere and pan) would remain unaltered after an experiment (after complete consumption of the sample) and that the baseline would remain unchanged. However, it was realized that the baseline measurements before and after an experiment were not the same for mixtures in which there were fueloxidizer reactions. In these cases, the system thermal absorption characteristics were being altered after an experiment, whereas, this effect was not present for single-component samples (decomposing but not reacting) such as with AN or NM. The effect was undone when the system was reheated and re-oxidized in air, in effect burning away any accumulated residues.

To reveal what was causing the change in the baseline, the following protocol was followed. In air at ambient pressure, the laser was used to oxidize the reactor sphere and pan surfaces (both having gold plated surfaces) by heating the sphere momentarily to about $773 \mathrm{~K}$. This procedure leaves a visually observed dark-grey copper oxide layer on the sphere and pan surfaces, and removes any residual materials on the copper surfaces. The chamber was closed, backfilled with nitrogen, and the pressure reduced to the operating condition. A thermogram was obtained for the baseline. The chamber was then opened and sample was placed on the sample thermocouple inside the reactor sphere. The chamber was again closed, backfilled with nitrogen ( 2 to 3 times), the pressure reduced, and a thermogram was obtained with the

sample. Note that the mass change due to evaporation under vacuum was considered negligible (NM has the highest vapor pressure, $3.65 \mathrm{kPa}$ at $293 \mathrm{~K}$, of the liquids used in this 
study and its mass change from its initial weighing until carrying out a measurement was considered small [10]). Assuming that chemical reactions go to completion and the sample is consumed completely, then one would expect the baseline (if repeated after the run) to remain unchanged as before the experiment, but this was not the case. The change in the baseline after the experiment was attributed to several possible reasons: 1) change in the reactor sphere inner surface due to fuel vaporization and deposition from a chemically reacting sample, or a change in the sphere outside-surface oxide layer, which would affect both the reactor and sample temperatures, and 2) sample residue remaining in the pan substrate (determined by reweighing the pan after the experiment) due to incomplete reaction of the sample, which would affect only the sample temperature. Thus, both the sample and reactor temperatures for the baseline thermogram could be influenced by a change in the system thermal characteristics.

To isolate these possible effects on the baseline measurement, another experiment was carried out as follows: 1) the sphere and pan were oxidized (i.e., observed to have a relatively darkgrey coating) and an initial baseline thermogram (before the sample run) was recorded followed by a repeated measurement; 2 ) sample (NMC) was then placed in the pan and the sample thermogram recorded followed by another baseline (after sample run) measurement; 3 ) the pan was then removed and the sphere re-oxidized, the pan was returned and another baseline (after) thermogram recorded; and finally, 4) both the sphere and pan were re-oxidized and another baseline thermogram acquired. Figure 3A presents for NMC both the reactor and sample temperatures for the initial baseline (before), sample run, and baseline (after) thermograms. Note that the symbols are not presented for each data point for clarity; a straight line is drawn between data points. The figure indicates that the baseline (after) thermogram changed to a lower temperature (for both the reactor and sample thermocouples) after the sample run, and did not reproduce the baseline (before) thermogram. The modification in the baseline appeared to be associated with a change in the surface absorption behavior of both the sphere surface and pan.

The baseline (before) thermogram for both the reactor and sample temperatures was repeated once, as presented in Fig. 3B. The result indicates a small temperature change to lower values after the measurement was repeated once (expecting no change). Observation of both the sphere outer and inner surfaces after the repeated run indicated that the smooth dark-grey copper (II) oxide ( $\mathrm{CuO})$ layer changed to that of a flaky reddish copper (I) oxide $\left(\mathrm{Cu}_{2} \mathrm{O}\right)$ appearance (see insert in Fig. 3B). Formation of CuO occurs between $673 \mathrm{~K}$ and $973 \mathrm{~K}$ with $\mathrm{Cu}_{2} \mathrm{O}$ appearing at higher temperatures $[32,33]$. As expected, a change in the sphere characteristics affected the thermal response of both thermocouples. When the sphere was reoxidized to the dark-grey $\mathrm{CuO}$ appearance and the baseline re-recorded, the baseline thermograms for both the reactor and sample temperatures are essentially the same except after reaching the steady-state temperature (see Fig. 3C). This small difference was again attributed to the changed sphere surface characteristics at temperatures above $973 \mathrm{~K}$. This 
result indicates that the sphere surface needs to be conditioned (by re-oxidizing the sphere and pan) before any experimental run so that the baseline thermogram is similar to the initial thermogram.

The effect of the pan residue was isolated by measuring the baseline after removing the pan and re-oxidizing only the sphere, and then recording another baseline after re-oxidizing both the sphere and pan. The results presented in Fig. 3D for both the reactor and sample temperatures again indicate that the pan with residue had lower temperatures than the reoxidized pan. It is also noted that the reactor temperature for both cases are different when expected to be similar after re-oxidation of the sphere. The reason for the difference is that the sphere for the residue-pan case was re-oxidized once while the oxidized-sphere/pan case was repeated twice, indicating that re-oxidation process of the former case was insufficient to return the surface to the original condition. This result indicates that the baseline after reoxidation needs to be checked and compared to the initial baseline thermogram, and one cannot rely solely on observation of the sphere surface. One concludes from these results that the sphere surface must be reheated (to re-establish the sphere CuO layer and remove any residue) using the same protocol before each run (regardless if a baseline or sample measurement). Also, it appears that the appropriate baseline thermogram to use is the initial one and that care must be taken to avoid systemic changes.

Regarding the sample thermogram (and correspondingly the sample derivative profile), a change in the baseline appears to reduce the heating rate associated with the sample thermogram, but the sample derivative profile should retain its features. This is analogous to changing the laser power, as discussed in the next section. In addition, during an experiment the ANF mixture is present inside the pan until consumed at the end of the reactions (residue cannot be present until completion of reactions). So by definition any remaining residue exists at the end of the run after release of all thermal energy by the ANF mixture. Also, reoxidization of the reactor sphere and pan (removing any sample residue, reforming the $\mathrm{CuO}$ layer, and keeping the experimental protocol unchanged for each measurement) returns the baseline back to near its original state, indicating the absence of fuel/pan reactions that permanently change the pan thermal behavior. Pan residue effects possibly could be minimized by reducing sample mass; however, there are mass effects to consider, as discussed in the next section.

Results confirm that fuel/oxidizer reactions are confined exclusively to the pan (i.e., without condensable vapor deposition and reaction of gaseous byproducts on the sphere inner surface. This is exemplified in Fig. 4, which presents the sample thermogram and sample derivative profile for ANCNM. The sample temperature is affected by the chemical reaction (as indicated by the dip in the thermogram at about 29 s, $590 \mathrm{~K}$ ) while the reactor temperature (being higher than that of the sample temperature due to direct surface heating by the laser) is essentially featureless for both the sample thermogram and sample derivative profile. This result indicates 
that the occurrence of chemical reaction influences the sample temperature (thermocouple at center of the reactor sphere) and not the reactor temperature (thermocouple flush with the sphere inner surface). Thus, the reactor temperature after a sample run should remain unchanged from the baseline reactor temperature if no alteration occurs in the copper oxide layer on the sphere surface.

\subsection{Sensitivity of the thermograms to sample mass and laser power}

The effect of sample mass and heating rate have been investigated in earlier investigations for AN [10] and ANFO (aging) [25]. An example of changing the sample mass is given in Fig. 5 for ANC and ANCNM. The figure presents the sample thermogram and sample derivative profile for each ANF mixture. For ANC, Figs. 5A and 5B compare results for masses of $2.5 \mathrm{mg}$ and 6.3 $\mathrm{mg}$. The mass of $\mathrm{C}_{\mathrm{TP}}$ was $1.0 \mathrm{mg}$ for each case and the pan substrate mass was $36.5 \mathrm{mg}$. The figure indicates that the lower mass developed less features in the sample derivative profile (Fig. 5B) than the larger mass for sample temperatures between $500 \mathrm{~K}$ and $900 \mathrm{~K}$, although not apparent from the sample thermogram (Fig. 5A). There is an unexplained feature for the 2.5 mg sample at around $950 \mathrm{~K}$, which is not present in the $6.3 \mathrm{mg}$ mass profile and may be attributed to consumption of the smaller mass. This result indicates that the thermograms are sensitive to the initial mass for binary mixtures and a minimum mass is required for detection of signature features. Similarly for the tri-component mixture ANCNM (Figs. 5C and 5D), the features are altered significantly when the mass changes from $5.7 \mathrm{mg}(2.0 \mathrm{mg}$ for AN, $1.0 \mathrm{mg}$ for

$\mathrm{C}_{\mathrm{TP}}$,

and

$2.7 \mathrm{mg}$ for $\mathrm{NM}$ ) to $6.7 \mathrm{mg}$ (3.2 $\mathrm{mg}$ for $\mathrm{AN}, 1.0 \mathrm{mg}$ for $\mathrm{C}_{\mathrm{TP}}$, and $2.5 \mathrm{mg}$ for $\mathrm{NM}$ ) - essentially, the mass for AN was increased by about $18.5 \%$. The signature features formed in the sample thermogram and sample derivative profile are more prominent for the heavier mass. Thus, although the peak intensities change, the association of the overall signature pattern with a particular ANF mixture composition does not change.

An example of changing the laser power is presented in Fig. 6 for the NMC thermogram and derivative profile. For this case, the heating rate changed from about $38 \mathrm{k} / \mathrm{s}$ to $59 \mathrm{~K} / \mathrm{s}$ (as defined earlier), an increase of about $55 \%$. The figure illustrates that in addition to increasing the heating rates and maximum achieved temperatures, the signature features are preserved, as denoted by the arrows between the two sample derivative profiles (Fig. 6B). For this case each mass was sufficient to establish signature features, while the magnitude of each peak changed with laser power. This case also provides an example of the reproducibility of the thermogram derivative profile features.

For cases with lower heating rates or smaller mass, the sample derivative profile may be devoid of features, as illustrated for AN in Ref. [10] Figs. 8 and 9). The fact that higher heating rates are required to detect derivative-profile features for several samples (see Table 1) indicates that detection of the sample thermal behavior is dependent on reaching steady-state 
temperature rapidly before decomposition of the mixture volatile components. At lower heating rates, one may speculate that either not enough energy was available for initiation of chemical reactions or volatile components evaporated before attaining temperatures associated with reaction of the sample, resulting in featureless profiles.

\subsection{Effect of fuel type on ANF mixture signature features}

Ammonium nitrate is the most commonly used oxidizer for energetic mixtures, while a variety of substances (depending on availability) are used as the fuel. In an earlier study [25], NM (single-component liquid fuel) was mixed with AN (single-component solid oxidizer) and the thermal signatures for the mixture ANNM were obtained under a variety of conditions. In another study [20], NM was saturated in nonreactive activated carbon and the thermal behavior reported for isolated NM. Figure 7 reproduces typical sample thermograms and sample derivative profiles for AN, NM, and the mixture ANNM. Note that the earlier NM results were obtained with a slower data acquisition system (with a data rate of 1 reading $\cdot \mathrm{s}^{-1}$ ) than the current arrangement and thus the profiles are noisier. Comparing the individual-component thermograms (Figs. 7A and 7C) to that of the mixture (Fig. 7E) indicates an endothermic behavior for the AN and ANNM thermograms for the reported heating rates [34], while NM was exothermic (Fig. 7C) for sample temperatures above $400 \mathrm{~K}$ (having a boiling point of $374 \mathrm{~K}$ [26]). The similar thermal behavior of ANNM and $A N$ is indicative of the fact that $A N$ is the dominant constituent. The features of the AN and ANNM derivative profiles are similar (Figs. 7B and 7F). At a sample temperature of about $480 \mathrm{~K}$, the sample derivative decreases for both cases, while the baseline increases to its maximum value (inflexion point in the sample thermogram). The decrease in the AN derivative is attributed to thermal decomposition of the solid AN at temperatures of between $480 \mathrm{~K}$ and $560 \mathrm{~K}$ [35]. The more significant decrease in the ANNM derivative is attributed also to NM evaporation. At around $530 \mathrm{~K}$, the AN and ANNM derivative increases due to AN consumption and a return of the thermogram to that of the baseline (see Fig. 7B). The higher-temperature maximum derivative for ANNM (Fig. 7F) generally reaches a larger value of $d T / d t$ than the lower-temperature maximum derivative, while the two peaks are comparable for AN. This larger higher-temperature peak (above the baseline value) is attributed to the reactive behavior of NM at sample temperatures above $550 \mathrm{~K}$ (see Fig. 7C and 7D). The sample temperature associated with each maximum and minimum derivative is higher for the AN case (as compared to ANNM). For example, the sample temperature associated with the derivative minima for ANNM (Fig. 7F) and AN (Fig. 7B) are $535 \mathrm{~K}$ and $563 \mathrm{~K}$, respectively. This result is attributed to the fact that more energy (i.e., higher temperatures) is required to decompose the single constituent $A N$, whereas the $N M$ in the mixture assists in decomposition of ANNM.

Figure 8 presents the sample thermograms and sample derivative profiles for the three liquid fuels DF (from Ref. [25]), $K_{1}$, and EG, which were mixed with AN (i.e., all referred to ANFO in the literature). The sample thermograms indicate that the ANDF (Fig. 8A) is essentially exothermic 
(with a small endotherm at about $500 \mathrm{~K}$ to $600 \mathrm{~K}$ ), while ANK and ANEG (Figs. 8C and 8E) are endothermic. The ANDF derivative profile (Fig. 8B) has similar features to that of ANNM but with the minimum derivative at about $500 \mathrm{~K}$. The ANK derivative profile (Fig. 8D) also shows initiation of a similar sample derivative profile with the minimum at about $540 \mathrm{~K}$. Note that the ANDF and ANK thermograms (or any AN/fuel mixture) will have more of an endothermic behavior (similar to AN) as the fuel component vaporizes with time. Also note that the ANDF and ANK thermograms reach the same sample temperature of about $900 \mathrm{~K}$, but the heating rates are significantly different (i.e., having the same laser power setting). The ANEG derivative profile

(Fig. 8F) is significantly different than the ANDF and ANK profiles, indicating the presence of two peaks at about $430 \mathrm{~K}$ and $580 \mathrm{~K}$. One would expect to use a higher laser power setting for lower volatility fuels, however as indicated in Table 1, this was not the case for EG (having a lower vapor pressure of $0.008 \mathrm{kPa}$ at $293 \mathrm{~K}$ [36], as compared to estimated values of $0.1 \mathrm{kPa}$ for $\mathrm{K}_{1}$ [37] and $0.3 \mathrm{kPa}$ for DF [38]). It is also noted that there is no relationship between the defined heating rate (i.e., peak in the baseline derivative profile) and the laser power setting (which influences the thermogram maximum sample temperature), indicating that the signature features are dependent on the ANF mixture thermal characteristics.

Figure 9 presents the sample thermograms and sample derivative profiles for the four solid fuels PJ, PW, S, and $\mathrm{C}_{\mathrm{TP}}$, which were mixed with AN (i.e., also defined as ANFO in the literature). The sample thermograms indicate that ANPJ, ANPW, and ANC are endothermic to different degrees, while the ANS thermogram exhibits an exothermic behavior at temperatures above $700 \mathrm{~K}$. The sample derivative profiles again are different from the other liquid-based ANFO profiles, but have the general feature of a minimum derivative somewhere in the middle of the profile, as found for AN. Also the sample temperatures associated with the derivative extrema are different for each case, indicating that the volatility and chemical reactivity are all different.

\subsection{Effect of oxidizer type on ANF mixture signature features}

For the cellulose fuel, $\mathrm{C}_{\mathrm{TP}}$, the oxidizer was changed from $\mathrm{AN}\left(\mathrm{NH}_{4} \mathrm{NO}_{3}\right)$ to $\mathrm{NM}\left(\mathrm{CH}_{3} \mathrm{NO}_{2}\right)$ and NB $\left(\mathrm{C}_{6} \mathrm{H}_{5} \mathrm{NO}_{2}\right)$. The compounds NM and NB were used as either a fuel or oxidizer, since the compounds contain both hydrocarbon and oxygen radical groups. Figure 10 presents the sample thermograms and sample derivative profiles for ANC, NMC, and NBC, along with the single-component signature for $\mathrm{C}_{\mathrm{TP}}$. Comparing the ANC mixture thermogram (Fig. $10 \mathrm{C}$ ) to the individual constituents $C_{T P}$ (Fig. 10A) and $A N$ (Fig. 7A), indicates that the behavior of $C_{T P}$ is exothermic at sample temperatures about $500 \mathrm{~K}$ and $\mathrm{AN}$ is endothermic, resulting in ANC being endothermic. This result suggests that the ANF mixture thermal behavior is dependent on the mass weighting, that is for the dominant constituent $A N$ (the $A N / C_{T P}$ mixture ratio being $90 \% / 10 \%$ ). Comparing the NM (Fig. 7C) and $C_{\text {TP }}$ thermograms with that of NMC (Fig. 10E) indicates that both constituents exothermic behavior while the mixture exhibits an endothermic behavior indicative of significant NM evaporation with increasing temperature. Similarly, the original NB data [20] were unavailable, but is also reported as being exothermic, 
while the NBC mixture thermogram (Fig. 10G) suggests significant NB evaporation. The ANC derivative profile (Fig. 10D) appears to have a similar contour to that of AN and ANNM (Figs. 7B and $7 F$, respectively), with its minimum at about $650 \mathrm{~K}$ (instead of AN minimum at about $550 \mathrm{~K}$ ). Again, the profile features being governed by the dominant mixture constituent (i.e., the AN oxidizer). Unlike ANC, the NMC sample derivative profile presents a series of peaks initiated at about

$400 \mathrm{~K}$, following the pattern indicated by NM in Fig. 7D, and the $C_{T P}$ profile in Fig. 10B. The NBC derivative profile formed features at a lower heating rate and was unlike the profile for both NMC and ANC.

\subsection{Effect of fuel type on three-component ANF mixture signature features}

Figure 11 presents the sample thermograms and sample derivative profiles for ANCNM and $A N C N B$, and are compared with the individual constituent results for $A N, N M$, and $C_{T P}$, as well as the two-component mixtures for ANC, NMC, and NBC. In general, the sample thermogram results for the three-component mixtures are not similar to the other signatures. Both mixtures exhibit an endothermic behavior consistent with the sample thermograms for AN, ANC, NMC, and NBC (the behavior was exothermic for NM and $\mathrm{C}_{\mathrm{TP}}$ ). Again, it appears that the endothermic behavior of the $A N$ was dominate over the contribution of $N M, N B$, and $C_{T P}$ in the tricomponent mixtures. Comparing sample derivative profiles, the ANCNM developed a sharp decrease in the derivative at about $550 \mathrm{~K}$ (Fig. 11B), which increases in magnitude with mass, as indicated

Fig. 5D. The NMC derivative profile (Fig. 10F) is characteristics of a series of small peaks, but with the addition of $A N$, the result is a dip similar to that of $A N$ in Fig. 7B. A decrease in the derivative is also obtained for ANCNB (Fig. 11D) at about $550 \mathrm{~K}$ (similar to that of AN), but the two peaks are different than for ANCNM. The ANCNM derivative profile is also unlike that for NMC

(Fig. 10H), indicating again that AN appears to be the dominant constituent. Thus, it is observed that the mixture signatures are individually different, depending on the mixture composition and constituent thermal behavior.

\section{Summary}

Thermal signatures for several ammonium nitrate/fuel mixtures (as recommended by collaborating forensic experts) were obtained and analyzed to demonstrate the capability of the laser-driven thermal reactor technique to provide detailed information for assisting in identifying precursor composition. This study addresses one aspect (i.e., to provide thermal signatures) of an overall strategy to statistically fuse signatures from a variety of analytical techniques and secondary forensics information. Results indicated that the reactor sphere has to be reheated (to re-establish the copper oxide layer and remove any residue) before a measurement to ensure repeatability. The measured thermograms were found to be different than the individual component fuels and oxidizers, as well as being different for each mixture, 
indicating sensitivity of the LDTR technique to ANF composition. The effects of the initial mass and laser power (once above the baseline threshold) did not appear to alter this finding. The thermal behavior of the ANF mixtures was found to be dependent on the constituent mass fraction, with each mixture component having its own thermal signature that is dependent on its chemical composition and thermal characteristics. More exhaustive measurements are needed under different operating conditions to better elucidate the effects of fuel volatility on the thermal signatures.

\section{Acknowledgements}

We gratefully acknowledge the support provided by the NIST Office of Law Enforcement Standards (project manager Mr. J.P. Jones). The authors would like to thank Dr. K. Yeager (FBI Laboratory) for his technical communications and discussions, and Dr. P. Chu for her helpful comments. 


\section{Nomenclature}

A sample geometric cross-sectional area $\left[\mathrm{m}^{2}\right]$

$c_{p}(T) \quad$ specific heat capacity $\left[\mathrm{J} \cdot \mathrm{g}^{-1} \cdot \mathrm{K}^{-1}\right]$

$(d T / d t) \quad$ sample temperature-time derivatives $\left[\mathrm{K} \cdot \mathrm{s}^{-1}\right]$

$F\left(T, T_{o}\right) \quad$ heat transfer term [W]

$I_{I} \quad$ intensity of the laser beam that heats the sample $\left[\mathrm{W} \cdot \mathrm{m}^{-2}\right.$ ]

$k \quad$ coverage factor

$m(t) \quad$ sample total initial mass [g]

$n \quad$ number of samples

$q(T) \quad$ specific heat release rate due to chemical reaction $\left[\mathrm{W} \cdot \mathrm{g}^{-1}\right.$ ]

$Q \quad$ total specific heat release (or absorption) $\left[\mathrm{kJ} \cdot \mathrm{g}^{-1}\right]$

$s \quad$ standard deviation

$t \quad$ time [s]

$T \quad$ temperature [K]

$T_{o} \quad$ steady-state sample temperature [K]

$u_{c} \quad$ combined uncertainty

$\underline{\text { Greek symbols }}$

$\beta(T) \quad$ absorptivity

$\lambda \quad$ laser wavelength [m]

$\Delta H \quad$ change in enthalpy $\left[\mathrm{kJ} \cdot \mathrm{g}^{-1}\right]$

$\Delta m \quad$ mass of the reactive portion of the sample, mass loss [g]

$\tau(T) \quad$ temperature-dependent relaxation time [s]

Subscripts

1,2

two different laser fluences

sas sample and substrate

so substrate only 


\section{References}

[1] Weapons Technical Intelligence (WTI) Improvised Explosive Device (IED) Lexicon, 4rd Edition, October 2012.

[2] ATF List of Explosive Materials, Federal Register, Vol. 76, No. 202, October 19, 2011.

[3] Afghanistan Route Clearance Handbook, US Army, Document No. 09-10, December, 2008.

[4] Improvised explosive precursors, FBI Bomb Data Center, Department of Justice, in Investigator Bulletin No. 2009-1, 2009.

[5] A. Miyake, H. Kobayashi, H. Echigoya, K. Kato, S. Kubota, Y. Wada, Y. Ogata, T. Ogawa, Detonation velocity and pressure of ammonium nitrate and activated carbon mixtures. Mater. Sci. Forum, 566 (2008) 107-112.

[6] A. Miyake, J. Echigoya, J. Kobayashi, K. Kato, S. Kubota, Y. Wada, Y. Ogata, T. Ogawa, Diameter effect on detonation velocity of ammonium nitrate and activated carbon mixtures. Mater. Sci. Forum, 566 (2008) 101-106.

[7] C. Hurley, V. Petr, S. Liu, J. Banker, Properties of alternatively fueled ammonium nitrate explosives. Proc. $9^{\text {th }}$ Int. Symp. on Rock Fragmentation by Blasting - FRAGBLAST9, Granada, Spain. 2009.

[8] D. Buczkowski, Ammonium nitrate - a treat of accidental explosion and terrorist attack, Chemik. 66:3 (2012) 231-234.

[9] D. Buczkowski, B. Zygmunt, Detonation properties of mixtures of ammonium nitrate based fertilizers and fuels. Cent. Eur. J. Energ. Mater. 8:2 (2011) 99-106.

[10] A. Nazarian, C. Presser, Forensic analysis methodology for thermal and chemical characterization of homemade explosives. Thermochim. Acta 576 (2014) 60-70.

[11] M. Kotrlý, I. Turková, Analysis of nonstandard and home-made explosives and post-blast residues in forensic practice. Chemical, Biological, Radiological, Nuclear, and Explosives (CBRNE) Sensing XV (ed. Augustus W. Fountain III), Proc. of SPIE, Vol. 9073, 2014.

[12] J. J. McShane, Issues in Explosives Residue Analysis: Identifying Techniques in explosive scene investigation. Part 10, 2012. http://www.thetruthaboutforensicscience.com/issues-inexplosives-residue-analysis-interpretation-of-data-in-explosive-scene-investigation/ 
[13] TWGFEX Laboratory Explosion Group: Standards and Protocols Committee, Recommended guidelines for forensic identification of post-blast explosive residues, Technical/Scientific Working Group for Fire and Explosions Analysis, 13 September 2009, Revision No. 8.

[14] F. Castanedo, A review of data fusion techniques. The Scientific World J., 2013 (2013) 1-19. http://dx.doi.org/10.1155/2013/704504.

[15] S. Challa, D. Koks, Bayesian and Dempster-Shafer fusion, Sadhana, Proc. Indian Academy of Sciences, 29:2 (2004) 145-174.

[16] A.P. Dempster, A generalization of Bayesian inference. J. R. Stat. Soc. Series B, 30 (1968) 205-247.

[17] G. Shafer, A Mathematical Theory of Evidence. Princeton Univ. Press, 1976.

[18] F. Cremer, K. Schutte, J.G.M. Schavemaker, and E. den Breejen, A comparison of decisionlevel sensor-fusion methods for anti-personnel landmine detection. Inf. Fusion, 2 (2001) 187208.

[19] A.J.R. Snell, Application of Differential Scanning Calorimetry to Characterize Thin Film Deposition Processes, Bachelor of Science in Chemical Engineering, Cleveland State University May, 2009. https://etd.ohiolink.edu/!etd.send file?accession=csu1280943337\&disposition= inline.

[20] A. Nazarian, C. Presser, Thermal and chemical kinetic characterization of multiphase and multicomponent substances by laser heating. Int. J. Heat Mass Transf., 51 (2008) 1365-1378.

[21] N.W., Piekiel, R.E. Cavicchi, M.R. Zachariah, Rapid-heating of energetic materials using a micro-differential scanning calorimeter. Thermochim. Acta, 521 (2011) 125-129.

[22] T.J. Janssen (Ed.), Explosive Materials Classification: Composition and Proper-ties, Nova Science, 2011, pp. 125-144.

[23] D. Maude, DSC Differential Scanning Calorimetry as used by AstraZeneca in Process Safety Evaluations, AstraZeneca, (2011). http://www.process-safety-lab.com/UploadFiles/201111 2118403931.pdf.

[24] G. Kubyshkina, Differential Scanning Calorimetry theoretical background, (2010). http://www.fs.uni-lj.si/mma bin.php?id=2010111100484104.

[25] A. Nazarian, C. Presser, Forensic methodology for the thermochemical characterization of ANNM and ANFO homemade explosives. Thermochim. Acta, 608 (2015) 65-75. 
[26] D.R. Lide (Ed.), Handbook of chemistry and physics. $95^{\text {th }}$ ed., CRC, Taylor and Francis Group, Florida, 2014-2015, pp. 3-414 and 12-206.

[27] B.N. Taylor, C.E. Kuyatt, Guidelines for evaluating and expressing the uncertainty of NIST measurement results. NIST Technical Note 1297, National Institute of Standards and Technology, Gaithersburg, MD, 1994.

[28] F.W. Sandstrom, M.G. Leone, K. Yeager, M. Miller, Diameter effects/critical diameter testing of fertilizer-grade ammonium nitrate based explosives in heavy confinement. Appendix E: Minimum booster size for AN based IEs - Safety Plan, Project No. J-FBI-05-173, 2006.

[29] Defense Intelligence Agency (DIA) Homemade Explosives Handbook, 2012

[30] http://www.chemistryislife.com/the-chemistry-of-toilet-paper; https://www.princeton.edu/ achaney/tmve/wiki100k/docs/Toilet paper.html.

[31] Personal communications.

[32] U. Nerle, M.K. Rabinal, Thermal oxidation of copper for favorable formation of cupric oxide (CuO) semiconductor. IOSR J. Appl. Phys., 5:1 (2013) 1-7.

[33] X. Jiang, T. Herricks, Y. Xia, CuO nanowires can be synthesized by heating copper substrates in air, Nano Lett., 2:12 (2002) 1333-1338.

[34] S. Chaturvedi, P.N. Dave, Review on thermal decomposition of ammonium nitrate. J. Energ. Mater., 31 (2013) 1-26.

[35] G. Feick , R.M. Hainer, On the thermal decomposition of ammonium nitrate. Steady-state reaction temperatures and reaction rate. J. Am. Chem. Soc., 76:22 (1954) 5860-5863. Also, http://www.societechimiquedefrance.fr/extras/Guiochon\%20VO/decompositionVO.htm.

[36] Ethylene Glycol Product Guide, MEGlobal (2008). http://www.meglobal.biz/media/ product guides/MEGlobal MEG.pdf.

[37] Material safety data sheet for kerosene, Sciencelab.com, Inc., 2013, CAS No. 8008-20-6.

[38] Material safety data sheet for automotive ultra-low diesel fuel, Mobil Oil Guam Inc., CAS No. 68334-30-5, 2011. 
Table 1: List of the investigated ANF mixtures, oxidizer/fuel mixture ratio, initial mass, and corresponding laser power used for each mixture. The cellulose was in the form of tissue paper. Except for NMC, NBC, ANCNM, and ANCNB, the mixture ratios are the stoichiometric values. The experiments were carried out under reduced pressure (4 $\mathrm{kPa})$ in a nitrogen environment.

\begin{tabular}{|c|c|c|c|c|}
\hline ANF Mixture & Oxidizer/Fuel & Initial Sample & Heating & Laser \\
& Mixture Ratio & Mass & Rate $^{\ddagger}$ & Power \\
& {$[\% / \%]$} & {$[\mathrm{mg}]$} & $\left.\mathrm{Ket}^{-1}\right]$ & Seting $^{\S}$ \\
\hline
\end{tabular}




\begin{tabular}{|c|c|c|c|c|c|}
\hline 1 & Ammonium nitrate/nitromethane & $71 / 29$ & $2.0 \pm 0.1$ & 60 & LS2 \\
\hline 2 & $\begin{array}{l}\text { Ammonium nitrate/No. } 2 \text { diesel fuel oil } \\
\text { (ANDF) }\end{array}$ & $94 / 6$ & $2.1 \pm 0.1$ & 60 & LS2 \\
\hline 3 & $\begin{array}{l}\text { Ammonium nitrate/kerosene } \\
(\mathrm{ANK})^{*}\end{array}$ & $94 / 6$ & $4.5 \pm 0.1$ & 29 & LS1 \\
\hline 4 & $\begin{array}{l}\text { Ammonium nitrate/ethylene glycol } \\
\text { (ANEG) }^{*}\end{array}$ & $87 / 13$ & $3.5 \pm 0.1$ & 33 & LS1 \\
\hline 5 & $\begin{array}{l}\text { Ammonium nitrate/petroleum jelly } \\
\text { (ANPJ) }\end{array}$ & $90 / 10$ & $3.6 \pm 0.1$ & 37 & LS1 \\
\hline 6 & Ammonium nitrate/paraffin wax & $90 / 10$ & $2.9 \pm 0.1$ & 32 & LS1 \\
\hline 7 & Ammonium nitrate/confectioner's sugar & $84 / 16$ & $3.2 \pm 0.1$ & 63 & LS2 \\
\hline 8 & & $90 / 10$ & $6.3 \pm 0.1$ & 76 & LS2 \\
\hline 9 & $\begin{array}{l}\text { (ANC) } \\
\text { Nitromethane/cellulose }\end{array}$ & saturated $^{\dagger}$ & $(4.2 / 1.0) \pm 0.1$ & 72 & LS2 \\
\hline 10 & (NMC) & saturated & $(3.3 / 0.8) \pm 0.1$ & 26 & LS1 \\
\hline 11 & (NBC) & 90/10/saturated & $\begin{array}{l}(3.2 / 1.0 / 2.5) \pm 0.1 \\
(2.0 / 1.0 / 2.7) \pm 0.1\end{array}$ & 26 & LS1 \\
\hline 12 & $\begin{array}{l}\text { Ammonium nitrate/cellulose/nitrobenzene } \\
\text { (ANCNB) }\end{array}$ & $90 / 10 /$ saturated & $(6.0 / 1.0 / 8.0) \pm 0.1$ & 82 & LS2 \\
\hline
\end{tabular}

*commonly referred to as ANFO.

'Saturated refers to soaking of the solid components with the liquid component, and thus the ratio will not be the stoichiometric value.

*The heating rate is defined by the maximum derivative of the baseline derivative profile (before the sample run), using the sample temperature.

${ }^{\S}$ LS1 is the lower value (total laser output power of about $69 \mathrm{~W}$ ) and LS2 is the higher value (output power of about $134 \mathrm{~W}$ ). 
Figures

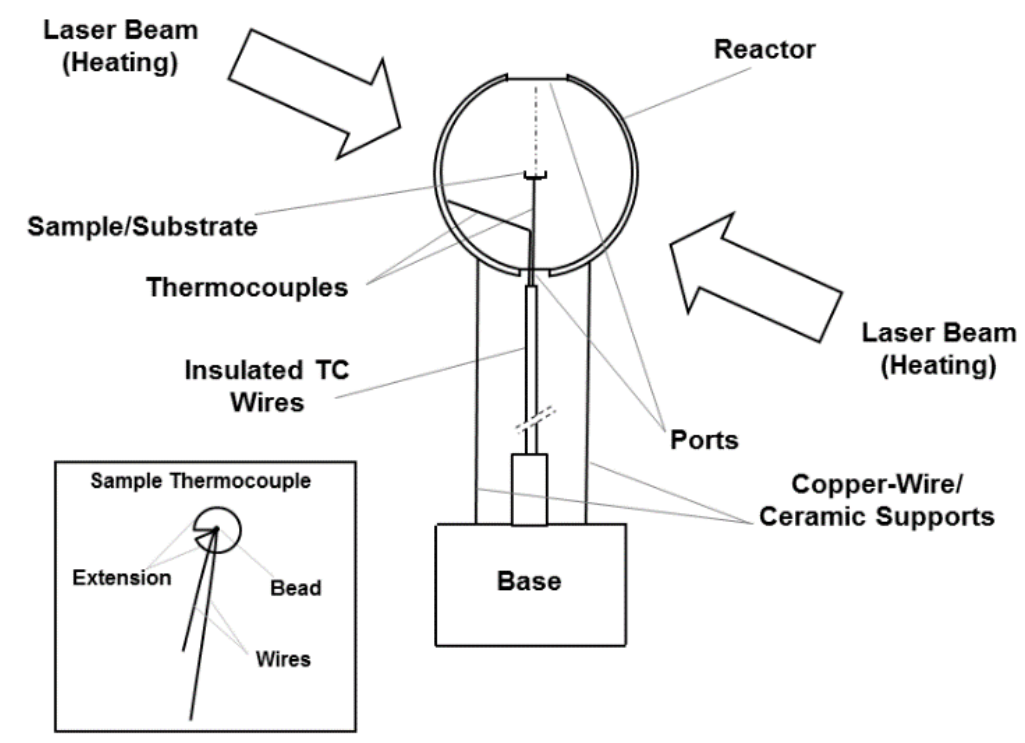

Figure 1. Schematic of the LDTR copper reactor sphere.

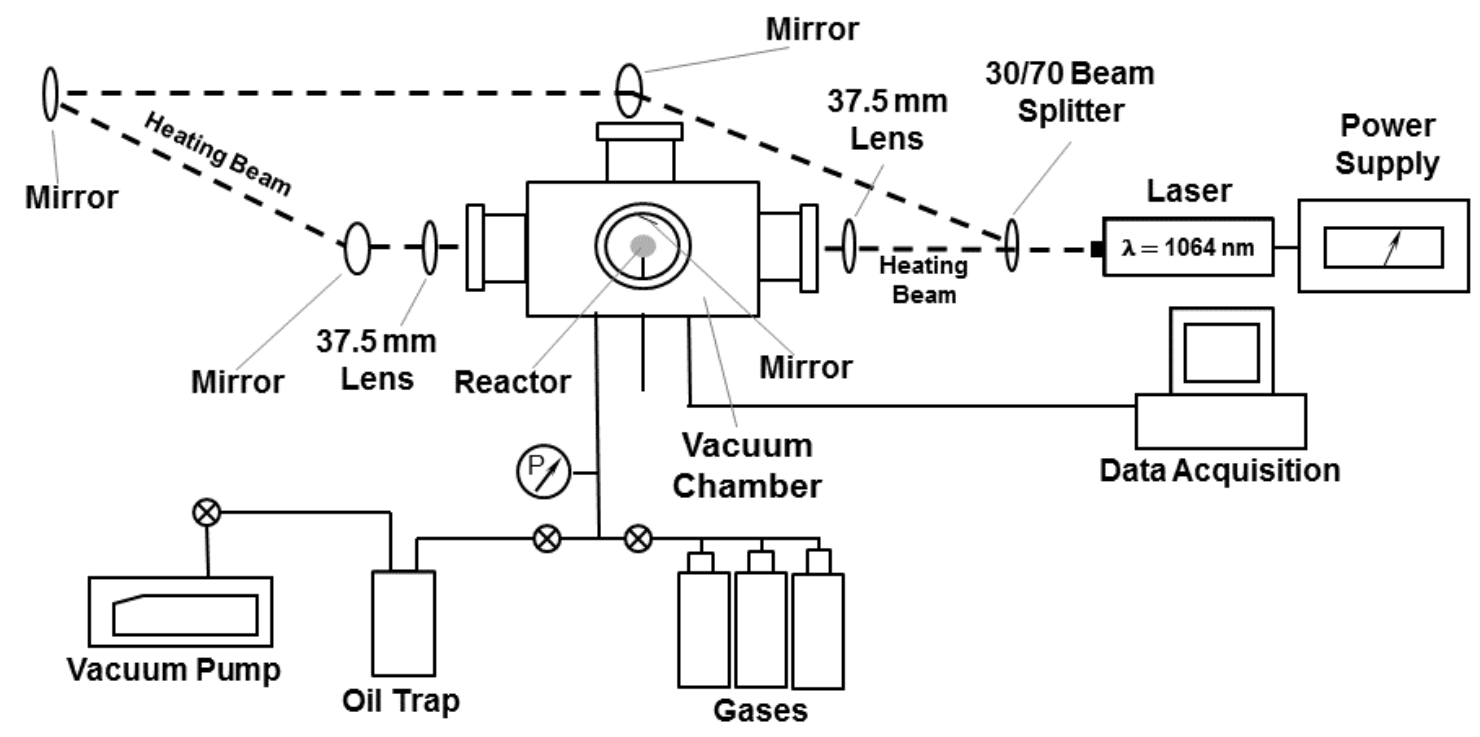

Figure 2. Schematic of the LDTR vacuum chamber and subsystems. 

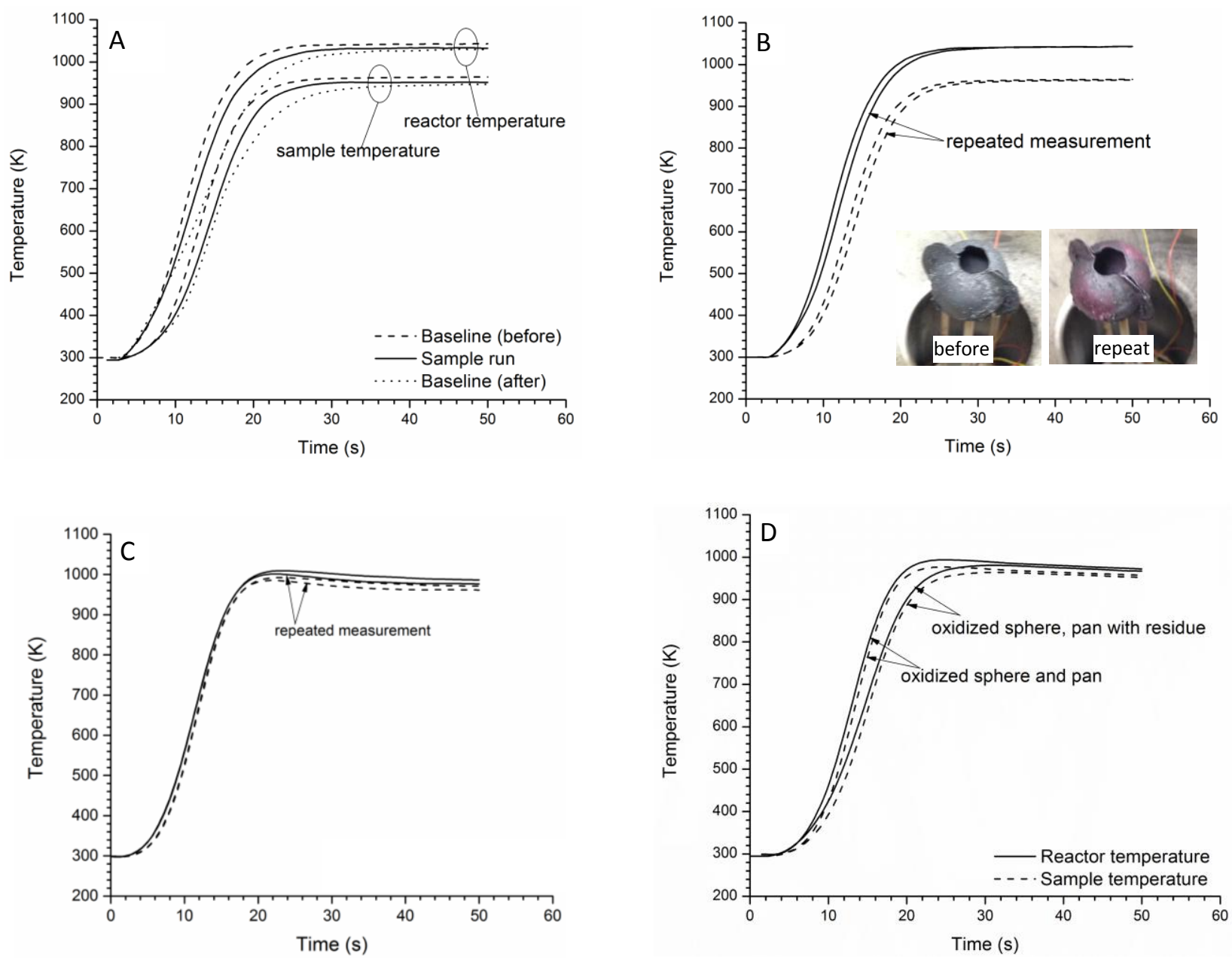

Figure 3. Variation in the baseline thermograms for the NMC mixture: comparison of $A$ ) baseline thermograms recorded before and after the sample thermogram, B) repeated baseline thermograms (before), C) repeated baseline thermograms (before) with sphere re-oxidation before each run, and D) the pan with and without residue. 

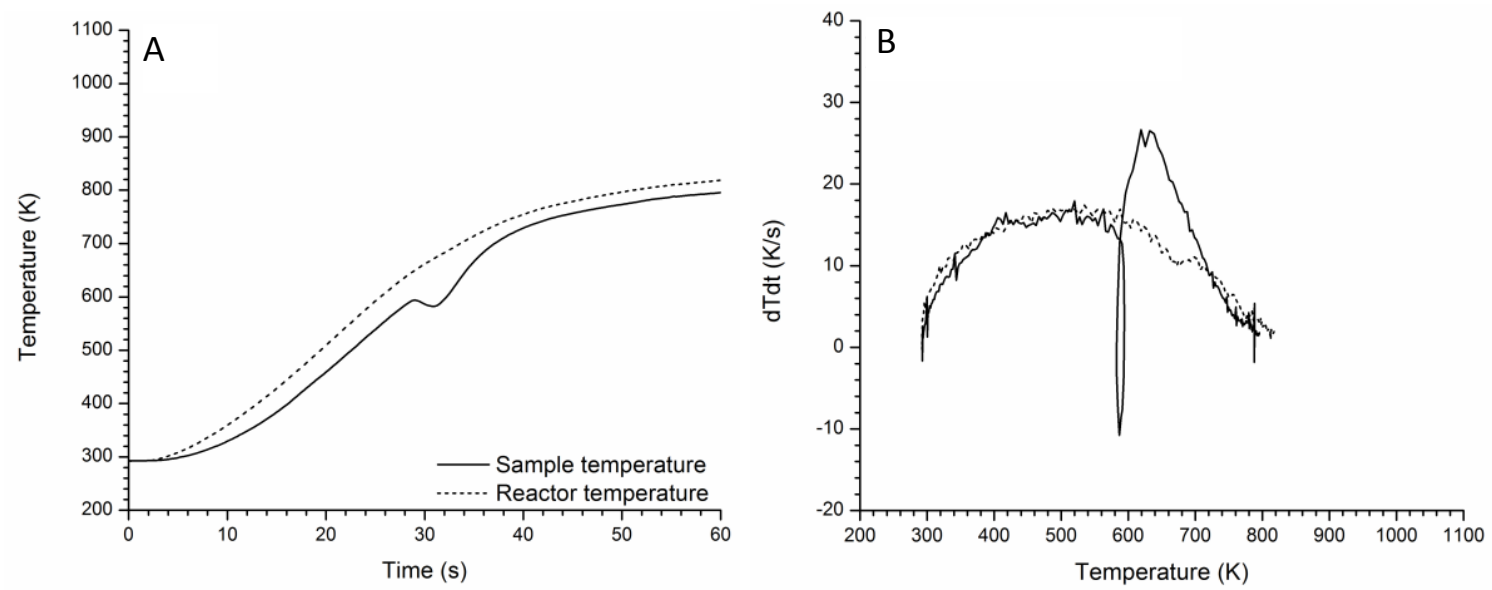

Figure 4: Comparison of the sample and reactor temperatures for the sample $A$ ) thermograms and B) derivative profiles, using ANCNM (mass of $6.7 \mathrm{mg} \pm 0.1 \mathrm{mg}$ ). 

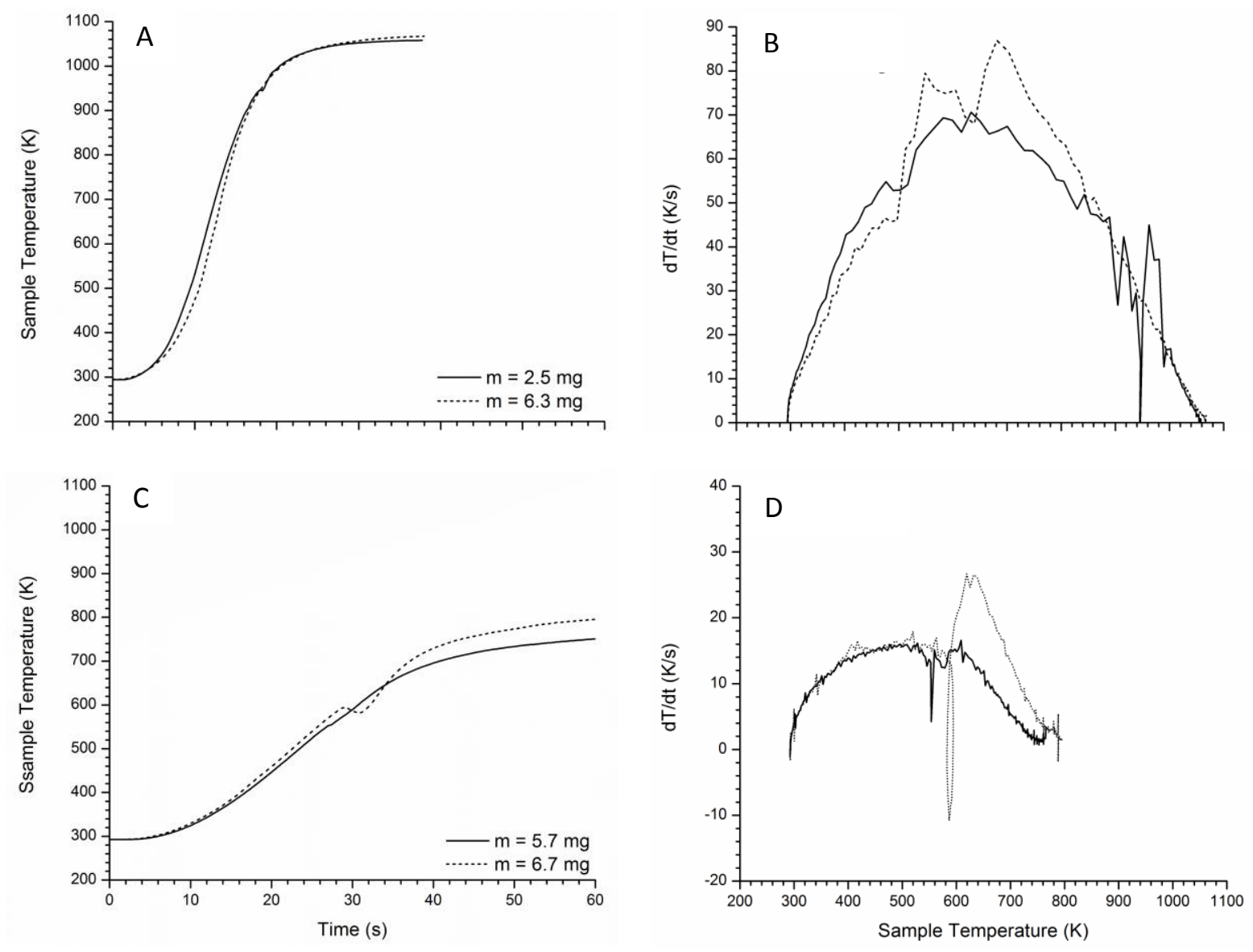

Figure 5. Effect of mass on the $A, C$ ) sample thermograms, and $B, D$ ) sample derivative profiles for ANC and ANCNM, respectively. 

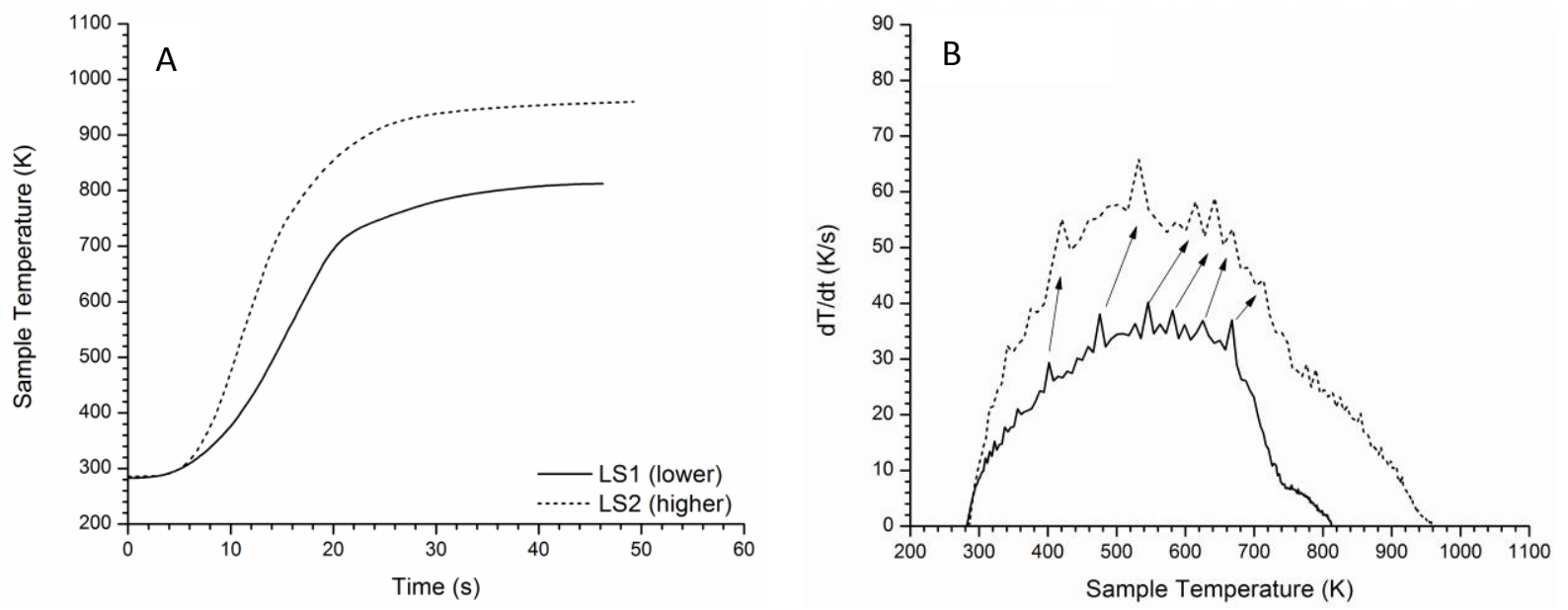

Figure 6. Effect of the laser power setting on the A) sample thermograms and B) sample derivative profiles for $\mathrm{NMC}$, having a $\mathrm{NM} / \mathrm{C}_{\mathrm{TP}}$ mass of $(4.2 / 1.0) \mathrm{mg} \pm 0.1 \mathrm{mg}$. 

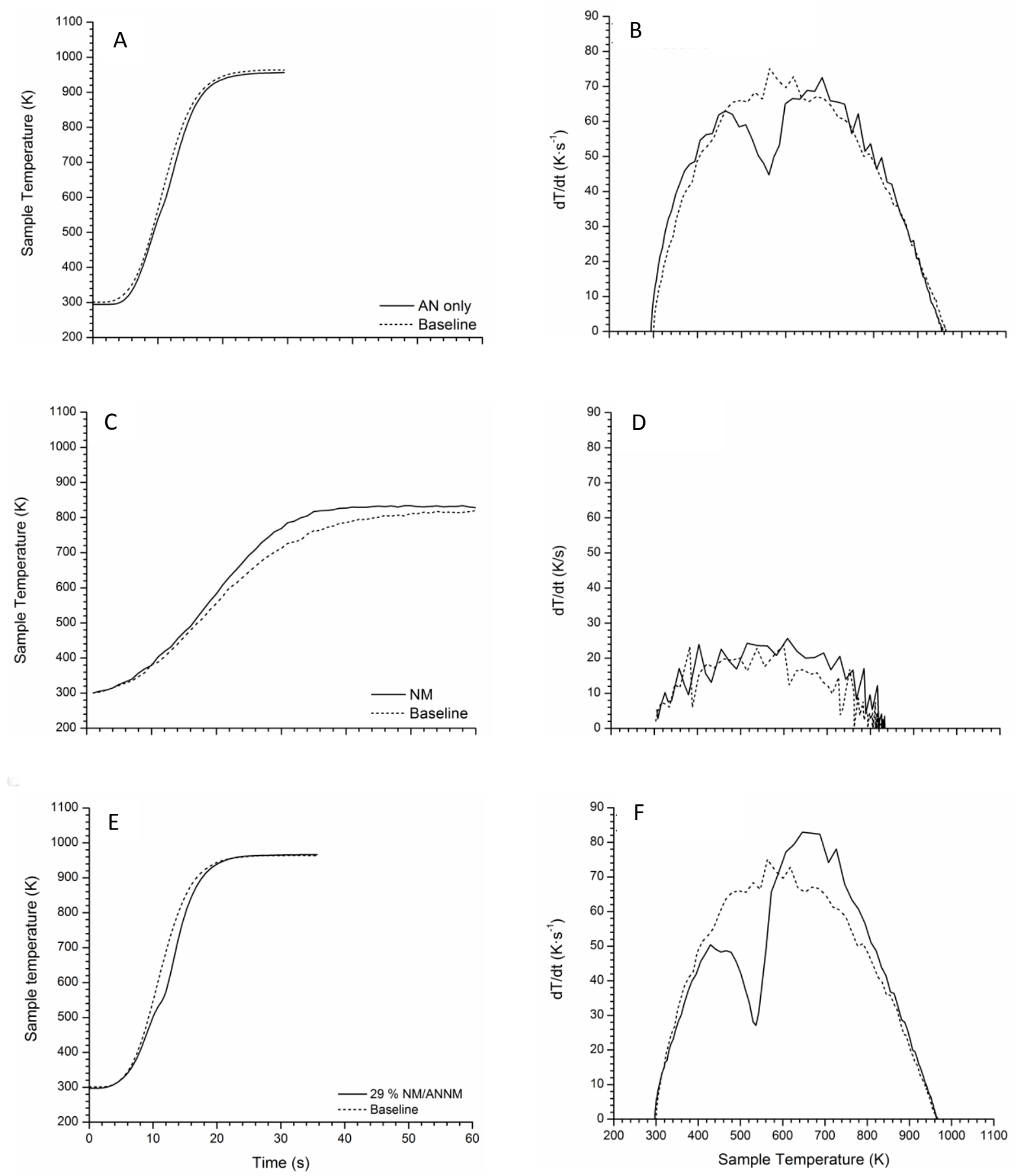

Figure 7. Comparison of the $A, C, E$ ) sample and baseline thermograms (sample temperature), and $B, D, F$ ) sample and baseline derivative profiles (sample temperature) for AN (singleconstituent oxidizer), NM (single-constituent fuel [10]), and the ANNM mixture, respectively. 

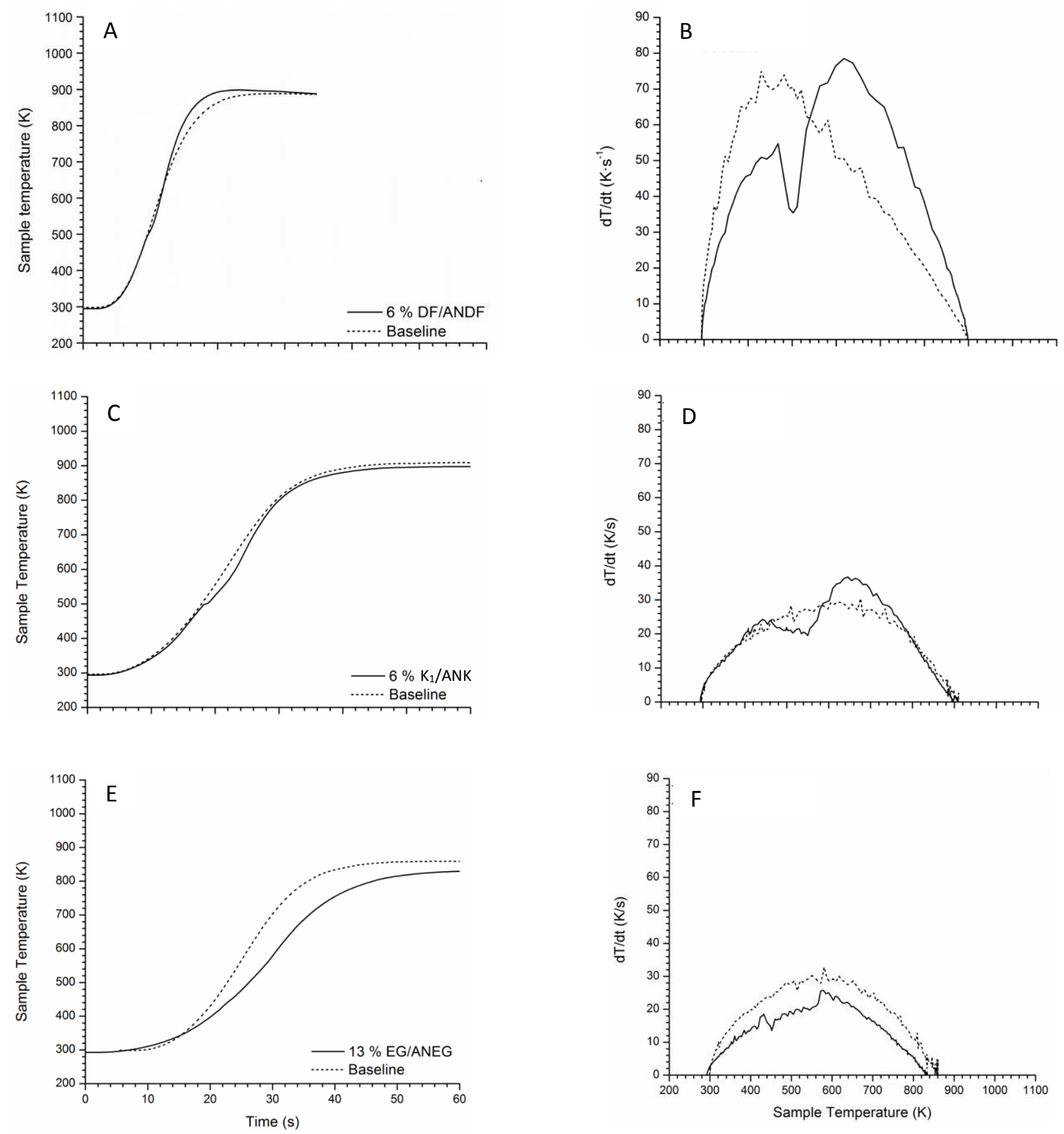

Figure 8. Comparison of the $A, C, E$ ) sample and baseline thermograms (sample temperature), and $B, D, F$ ) sample and baseline derivative profiles (sample temperature) for liquid-fueled ANFO (i.e., ANDF, ANK, and ANEG, respectively). 

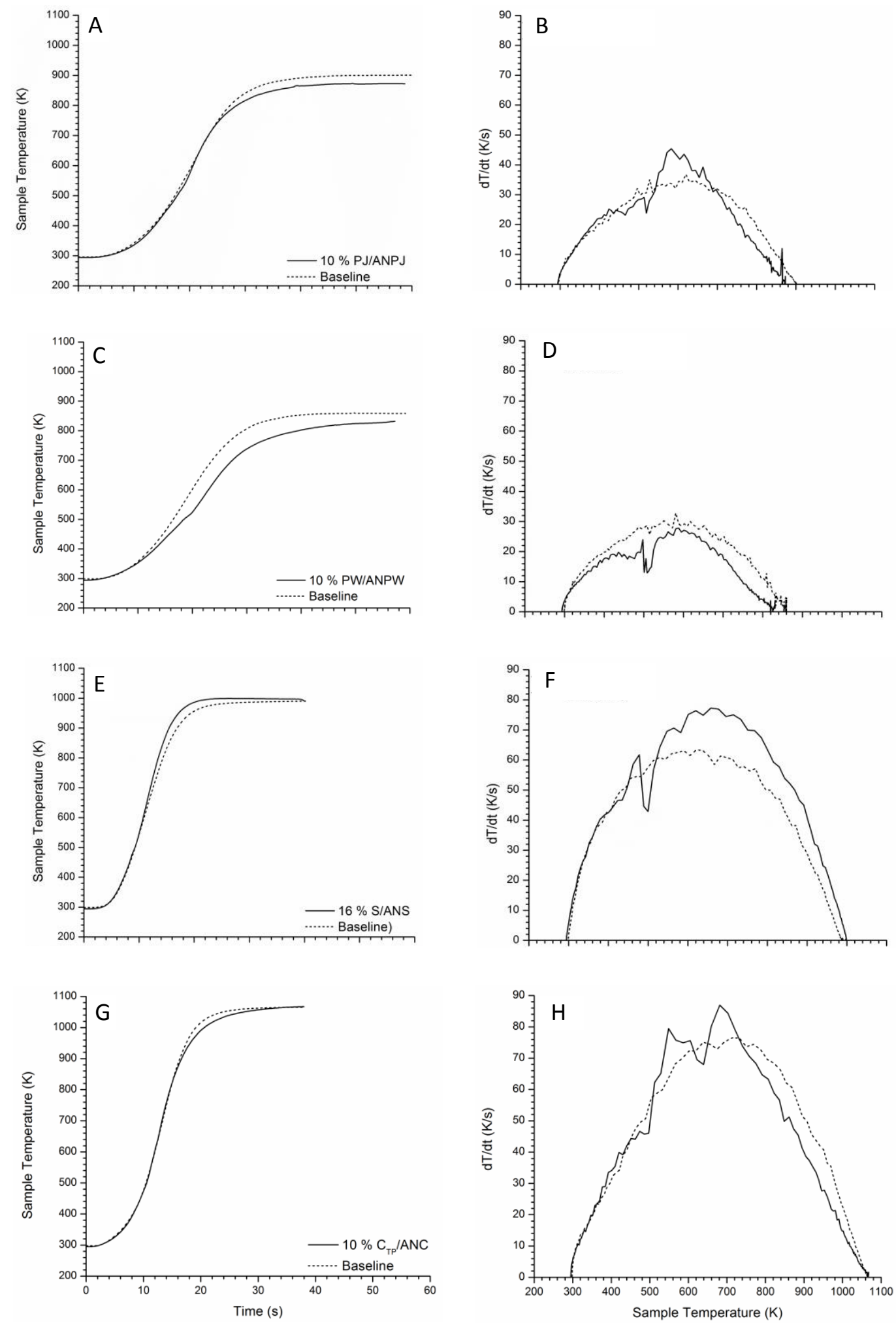

Figure 9. Comparison of the A, C, E, G) sample and baseline thermograms (sample temperature), and $B, D, F, H$ ) sample and baseline derivative profiles (sample temperature) for solid-fueled ANFO (i.e., ANPJ, ANPW, ANS, and ANC, respectively). 

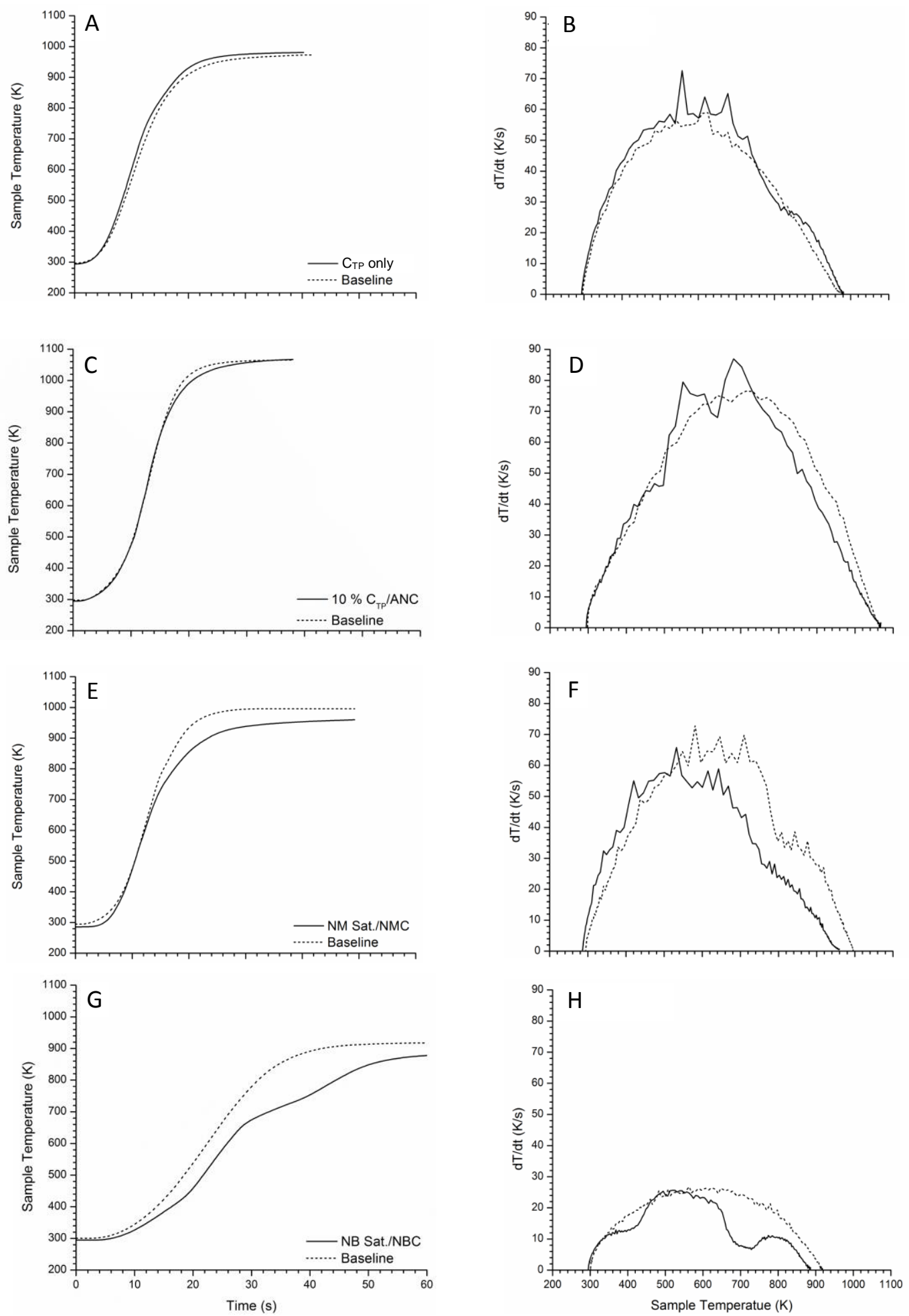

Figure 10. Comparison of the A, C, E, G) sample and baseline thermograms (sample temperature), and $B, D, F, H$ ) sample and baseline derivative profiles (sample temperature) for the single-component fuel $C(T P)$ and different oxidizers (i.e., AN, NM, and NB, respectively). 

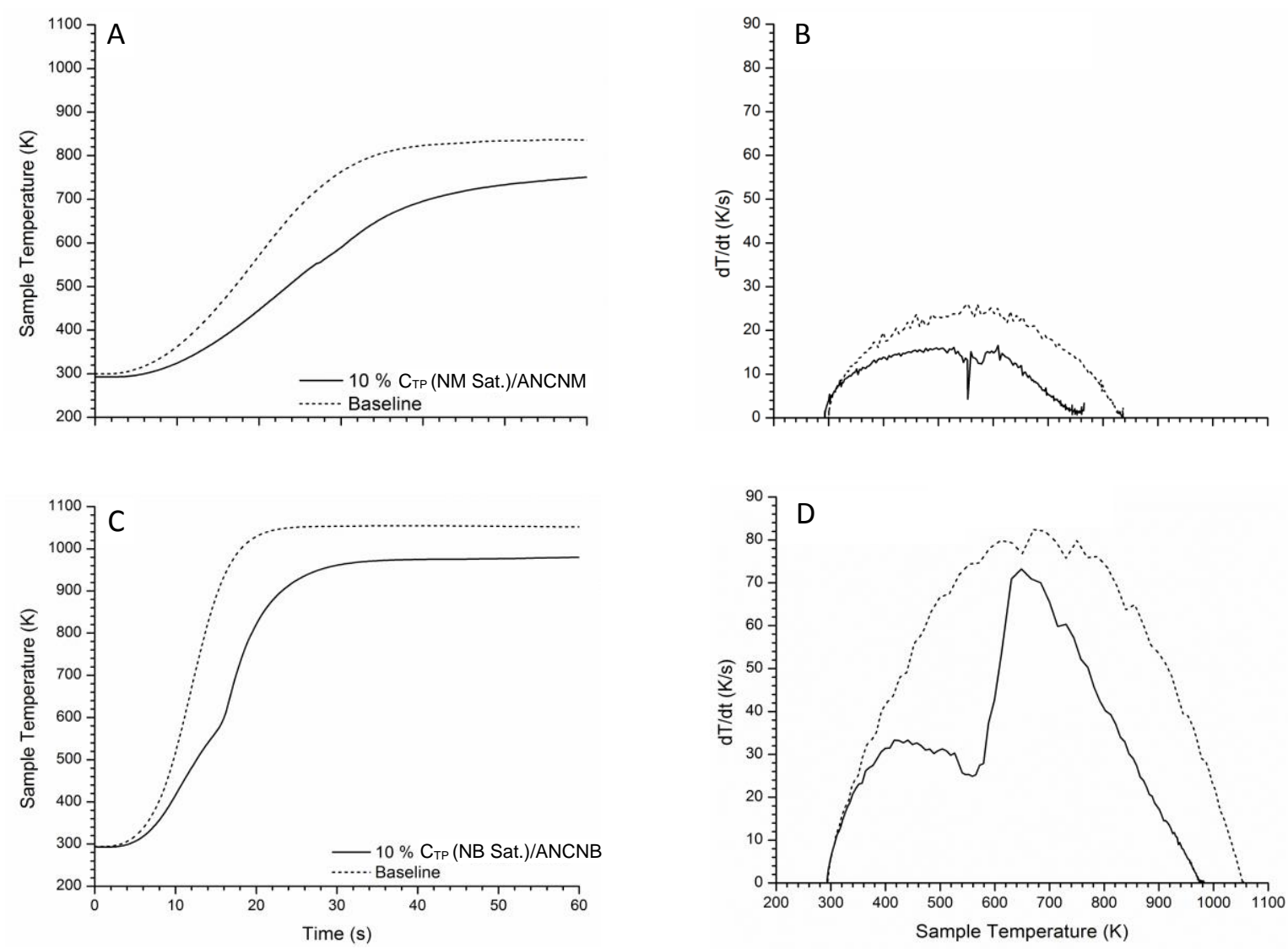

Figure 11. Comparison of the $A, C$ ) sample and baseline thermograms (sample temperature), and $B, D)$ sample and baseline derivative profiles (sample temperature) for tri-component HME with different liquid fuels (i.e., ANCNM and ANCNB, respectively). 